\title{
Spectral approach to homogenization of an elliptic operator periodic in some directions.
}

\author{
R. Bunoiu \\ LMAM, UMR 7122, Université de Metz et CNRS \\ Ile du Saulcy, F-57045 METZ Cedex 1, France \\ email: bunoiu@math.univ-metz.fr \\ G. Cardone \\ University of Sannio - Department of Engineering \\ Piazza Roma, 21 - 84100 Benevento, Italy \\ email: giuseppe.cardone@unisannio.it \\ T. Suslina \\ St. Petersburg State University, Department of Physics \\ Petrodvorets, Ul'yanovskaya 1, St. Petersburg 198504, Russia \\ email: suslina@list.ru
}

November 9, 2018

\begin{abstract}
The operator

$$
A_{\varepsilon}=D_{1} g_{1}\left(x_{1} / \varepsilon, x_{2}\right) D_{1}+D_{2} g_{2}\left(x_{1} / \varepsilon, x_{2}\right) D_{2}
$$

is considered in $L_{2}\left(\mathbb{R}^{2}\right)$, where $g_{j}\left(x_{1}, x_{2}\right), j=1,2$, are periodic in $x_{1}$ with period 1 , bounded and positive definite. Let function $Q\left(x_{1}, x_{2}\right)$ be bounded, positive definite and periodic in $x_{1}$ with period 1. Let $Q^{\varepsilon}\left(x_{1}, x_{2}\right)=Q\left(x_{1} / \varepsilon, x_{2}\right)$. The behavior of the operator $\left(A_{\varepsilon}+Q^{\varepsilon}\right)^{-1}$ as $\varepsilon \rightarrow 0$ is studied. It is proved that the operator $\left(A_{\varepsilon}+Q^{\varepsilon}\right)^{-1}$ tends to $\left(A^{0}+Q^{0}\right)^{-1}$ in the operator norm in $L_{2}\left(\mathbb{R}^{2}\right)$. Here $A^{0}$ is the effective operator whose coefficients depend only on $x_{2}, Q^{0}$ is the mean value of $Q$ in $x_{1}$. A sharp order estimate for the norm of the difference $\left(A_{\varepsilon}+Q^{\varepsilon}\right)^{-1}-\left(A^{0}+Q^{0}\right)^{-1}$ is obtained. The result is applied to homogenization of the Schrödinger operator with a singular potential periodic in one direction.
\end{abstract}

\section{Introduction}

\subsection{Spectral approach to homogenization problems}

Homogenization problems for periodic differential operators of mathematical physics are of significant interest both from theoretical point of view and for applications. A broad literature is devoted to homogenization problems. At the first place, the books [BeLP], $[\mathrm{Sa}], \mathrm{BaPa}], \mathrm{ZhKO}]$ should be mentioned.

Consider a typical homogenization problem. Let $\mathcal{A}_{\varepsilon}$ be a family of operators in $L_{2}\left(\mathbb{R}^{d}\right)$ given by

$$
\mathcal{A}_{\varepsilon}=-\operatorname{div} g(\mathbf{x} / \varepsilon) \nabla, \quad \varepsilon>0 .
$$

Here $g(\mathbf{x})$ is a positive definite and bounded $(d \times d)$-matrix-valued function. It is assumed that $g(\mathbf{x})$ is periodic with respect to some lattice $\Gamma \subset \mathbb{R}^{d}$. The problem is to study the behavior of the solution $u_{\varepsilon}$ of the equation

$$
\mathcal{A}_{\varepsilon} u_{\varepsilon}+u_{\varepsilon}=F, \quad F \in L_{2}\left(\mathbb{R}^{d}\right),
$$


for small $\varepsilon$. It turns out that $u_{\varepsilon}$ tends (in some sense) to $u_{0}$ as $\varepsilon \rightarrow 0$, where $u_{0}$ is the solution of the "homogenized" equation

$$
\mathcal{A}^{0} u_{0}+u_{0}=F \text {. }
$$

Here $\mathcal{A}^{0}=-\operatorname{div} g^{0} \nabla$, and $g^{0}$ is a constant positive matrix called the effective matrix. The operator $\mathcal{A}^{0}$ is called the effective operator. The character of convergence of $u_{\varepsilon}$ to $u_{0}$ and error estimates are studied.

A possible approach to homogenization of periodic differential operators in $\mathbb{R}^{d}$ consists in application of the Floquet-Bloch theory combining with methods of the analytic perturbation theory. In this connection, we mention the papers $\mathrm{Se}, \mathrm{Zh}$, $\mathrm{CoV}$. In a series of papers BSu1, BSu2, BSu3, BSu4, a new operator-theoretic approach to homogenization problems was suggested and developed. It was also based on the Floquet-Bloch decomposition for a periodic operator and applying methods of the analytic perturbation theory. The main idea of this approach was to study a homogenization procedure as a spectral threshold effect at the bottom of the spectrum of a periodic elliptic operator. In particular, this approach allowed the authors to prove the following error estimate:

$$
\left\|u_{\varepsilon}-u_{0}\right\|_{L_{2}\left(\mathbb{R}^{d}\right)} \leq C \varepsilon\|F\|_{L_{2}\left(\mathbb{R}^{d}\right)}
$$

see BSu1. This estimate is order-sharp and the constant $C$ is well controlled. Estimate (1.4) means that the resolvent $\left(\mathcal{A}_{\varepsilon}+I\right)^{-1}$ tends to $\left(\mathcal{A}^{0}+I\right)^{-1}$ in the operator $L_{2}$-norm, and

$$
\left\|\left(\mathcal{A}_{\varepsilon}+I\right)^{-1}-\left(\mathcal{A}^{0}+I\right)^{-1}\right\|_{L_{2}\left(\mathbb{R}^{d}\right) \rightarrow L_{2}\left(\mathbb{R}^{d}\right)} \leq C \varepsilon .
$$

Such estimates are called "operator error estimates"; they were obtained in [BSu1] for a wide class of matrix periodic differential operators.

Let us briefly discuss the method of the proof of estimate (1.5). By the scaling transformation, (1.5) is equivalent to the estimate

$$
\left\|\left(\mathcal{A}+\varepsilon^{2} I\right)^{-1}-\left(\mathcal{A}^{0}+\varepsilon^{2} I\right)^{-1}\right\|_{L_{2}\left(\mathbb{R}^{d}\right) \rightarrow L_{2}\left(\mathbb{R}^{d}\right)} \leq C \varepsilon^{-1} .
$$

Here $\mathcal{A}=-\operatorname{div} g(\mathbf{x}) \nabla$. The bottom of the spectrum of $\mathcal{A}$ is the point $\lambda=0$. It is natural that the behavior of the resolvent $\left(\mathcal{A}+\varepsilon^{2} I\right)^{-1}$ can be described in terms of the threshold characteristics of $\mathcal{A}$ (i. e., the spectral characteristics at the bottom of the spectrum). Applying the Floquet-Bloch theory, we decompose $\mathcal{A}$ in the direct integral of operators $\mathcal{A}(\mathbf{k})$ acting in $L_{2}(\Omega)$. Here $\Omega$ is the cell of the lattice $\Gamma$. The parameter $\mathbf{k} \in \mathbb{R}^{d}$ is called a quasimomentum. The operator $\mathcal{A}(\mathbf{k})$ is given by the differential expression $(\mathbf{D}+\mathbf{k})^{*} g(\mathbf{x})(\mathbf{D}+\mathbf{k})$ with periodic boundary conditions. Estimate (1.6) is equivalent to the estimate

$$
\left\|\left(\mathcal{A}(\mathbf{k})+\varepsilon^{2} I\right)^{-1}-\left(\mathcal{A}^{0}(\mathbf{k})+\varepsilon^{2} I\right)^{-1}\right\|_{L_{2}(\Omega) \rightarrow L_{2}(\Omega)} \leq C \varepsilon^{-1}
$$

which must be uniform in $\mathbf{k} \in \widetilde{\Omega}$. Here $\widetilde{\Omega}$ is the Brillouin zone of the dual lattice $\widetilde{\Gamma}$. The main part of investigation is the study of the operator family $\mathcal{A}(\mathbf{k})$ by means of the analytic perturbation theory. A crucial role is played by the spectral germ of the operator family $\mathcal{A}(\mathbf{k})$ at $\mathbf{k}=0$. The spectral germ is a finite rank selfadjoint operator which is defined in terms of the threshold characteristics of $\mathcal{A}$. It is possible to find a finite rank approximation for the resolvent $\left(\mathcal{A}(\mathbf{k})+\varepsilon^{2} I\right)^{-1}$ for small $\varepsilon$ in terms of the spectral germ. Next, it turns out that the family $\mathcal{A}^{0}(\mathbf{k})$ corresponding to the effective operator has the same spectral germ as the family $\mathcal{A}(\mathbf{k})$. This allows one to pass from approximation in terms of the germ to approximation in terms of the effective operator.

\subsection{Homogenization problems for operators periodic not in all directions}

Along with operators whose coefficients are periodic in all directions, it is interesting to study operators with coefficients that are periodic only in part of variables. Such problems are important for applications, in particular, in the theory of waveguides. In these problems, the coefficients of the effective operator depend on the "nonperiodic" variables, while homogenization is related to the "periodic" variables. Technically, the study of such problems is more difficult than the study of operators with coefficients that are periodic in all directions. 
In the paper $[\mathrm{Su}]$, the following elliptic operator in a strip $\Pi=\mathbb{R} \times(0, a)$ was considered:

$$
\mathcal{B}_{\varepsilon}=D_{1} g_{1}\left(\varepsilon^{-1} x_{1}, x_{2}\right) D_{1}+D_{2} g_{2}\left(\varepsilon^{-1} x_{1}, x_{2}\right) D_{2} .
$$

The coefficients $g_{1}, g_{2}$ were assumed to be positive definite, bounded and 1-periodic in $x_{1}$. Moreover, it was assumed that $g_{1}, g_{2}$ are periodic Lipschitz functions with respect to $x_{2}$. On the boundary $\partial \Pi$, periodic boundary conditions were posed. It was shown that there exists an "effective operator" $\mathcal{B}^{0}=$ $D_{1} g_{1}^{0}\left(x_{2}\right) D_{1}+D_{2} g_{2}^{0}\left(x_{2}\right) D_{2}$ (also with periodic boundary conditions on $\partial \Pi$ ) such that

$$
\left\|\left(\mathcal{B}_{\varepsilon}+I\right)^{-1}-\left(\mathcal{B}^{0}+I\right)^{-1}\right\|_{L_{2}(\Pi) \rightarrow L_{2}(\Pi)} \leq C \varepsilon .
$$

As before, the scaling transformation is used and the Floquet decomposition (in $x_{1}$ ) is applied. The quasimomentum $k$ is one-dimensional. Then the problem reduces to the study of the operator family

$$
\mathcal{B}(k, \varepsilon)=\mathcal{B}_{1}(k)+\varepsilon^{2} \mathcal{B}_{2},
$$

acting in $L_{2}(\Omega)$, where $\Omega=(0,1) \times(0, a)$. Here $\mathcal{B}_{1}(k)$ is given by the expression $\left(D_{1}+k\right) g_{1}(\mathbf{x})\left(D_{1}+k\right)$ and $\mathcal{B}_{2}=D_{2} g_{2}(\mathbf{x}) D_{2}$; the boundary conditions are periodic in both variables. The method of BSu1] can be applied to the operator $\mathcal{B}_{1}(k)$ "layerwise". This allows to introduce the spectral germ for the family $\mathcal{B}_{1}(k)$ and to obtain approximation for $\mathcal{B}_{1}(k)$ in terms of the germ. Main technical difficulties are related to adding the unbounded "perturbation" term $\varepsilon \mathcal{B}_{2}$ to $\mathcal{B}_{1}(k)$ and approximating the operator $\left(\mathcal{B}(k, \varepsilon)+\varepsilon^{2} I\right)^{-1}$.

\subsection{Main goal and main results}

In the present paper, we study an elliptic operator

$$
A_{\varepsilon}=D_{1} g_{1}\left(\varepsilon^{-1} x_{1}, x_{2}\right) D_{1}+D_{2} g_{2}\left(\varepsilon^{-1} x_{1}, x_{2}\right) D_{2}, \quad \varepsilon>0,
$$

acting in $L_{2}\left(\mathbb{R}^{2}\right)$. The coefficients $g_{j}\left(x_{1}, x_{2}\right), j=1,2$, are assumed to be bounded, Lipschitz in $x_{2}$, positive definite and 1-periodic in $x_{1}$. Next, let $Q(\mathbf{x})$ be a positive definite and bounded function in $\mathbb{R}^{2}$. It is assumed that $Q$ is 1-periodic in $x_{1}$ and Lipschitz in $x_{2}$. We denote $Q^{\varepsilon}(\mathbf{x})=Q\left(\varepsilon^{-1} x_{1}, x_{2}\right)$.

Our main goal is to find an approximation in the operator norm in $L_{2}\left(\mathbb{R}^{2}\right)$ for the generalized resolvent $\left(A_{\varepsilon}+Q^{\varepsilon}\right)^{-1}$ of the operator (1.9).

We construct the effective operator

$$
A^{0}=D_{1} g_{1}^{0}\left(x_{2}\right) D_{1}+D_{2} g_{2}^{0}\left(x_{2}\right) D_{2}
$$

with the effective coefficients depending only on $x_{2}$ (see (2.10), (2.11) $)$. By $Q^{0}\left(x_{2}\right)$ we denote the mean value of $Q\left(x_{1}, x_{2}\right)$ in $x_{1}$.

The main result of the paper is the following estimate

$$
\left\|\left(A_{\varepsilon}+Q^{\varepsilon}\right)^{-1}-\left(A^{0}+Q^{0}\right)^{-1}\right\|_{L_{2}\left(\mathbb{R}^{2}\right) \rightarrow L_{2}\left(\mathbb{R}^{2}\right)} \leq C \varepsilon .
$$

By the scaling transformation, the question is reduced to the study of the operator family $\left(A(\varepsilon)+\varepsilon^{2} Q\right)^{-1}$, where the operator

$$
A(\varepsilon)=D_{1} g_{1}\left(x_{1}, x_{2}\right) D_{1}+\varepsilon^{2} D_{2} g_{2}\left(x_{1}, x_{2}\right) D_{2}
$$

contains small factor $\varepsilon^{2}$ standing at the second order operator $D_{2} g_{2} D_{2}$. This is the main difficulty. We apply the Floquet-Bloch decomposition (in $x_{1}$ ) and decompose the operator (1.11) in the direct integral of operators

$$
A(k, \varepsilon)=A_{1}(k)+\varepsilon^{2} A_{2},
$$

acting in $L_{2}(\Omega)$. Now the domain $\Omega$ is unbounded: $\Omega=(0,1) \times \mathbb{R}$. The operator $A_{1}(k)$ is given by the expression $\left(D_{1}+k\right) g_{1}(\mathbf{x})\left(D_{1}+k\right)$ with periodic boundary conditions on $\partial \Omega$, and $A_{2}=D_{2} g_{2}(\mathbf{x}) D_{2}$. We apply method of [BSu1] to the operator $A_{1}(k)$ "layerwise" (for each fixed $x_{2}$ ), and define the spectral 
germ of the family $A_{1}(k)$ at $k=0$. Now the germ is not a finite rank operator. It is possible to approximate the part of $A_{1}(k)$ near the bottom of the spectrum in terms of the germ. Main difficulties are related to taking the term $\varepsilon^{2} A_{2}$ into account.

The essential differences of the problem studied here from the problem studied before in $[\mathrm{Su}]$ is that $\Omega$ is unbounded domain and that we study the generalized resolvent of $A_{\varepsilon}$ (instead of the ordinary resolvent). We develop some technical tools suggested in $\underline{\mathrm{Su}}$ and adapt them for the case of unbounded domain $\Omega$ and generalized resolvent.

Main results can be generalized for the case of arbitrary dimension without any new technical difficulties. We give formulations of the corresponding results (see Section 10). In the main exposition, we consider the case $d=2$ only for the sake of simplicity and clearness.

Our general result can be applied to homogenization of the Schrödinger operator with a singular potential periodic in one direction and non-periodic in another direction; see Section 9. This operator arises in the model of a "soft waveguide" (the spectral properties of such operators were studied in [FK]). We emphasize that for application to the Schrödinger operator we need to study in advance a generalized resolvent of $A_{\varepsilon}$ (but not the ordinary resolvent).

\subsection{Plan of exposition}

In Section 2 we give the precise statement of the problem and formulate the main result (Theorem 1). In Section 3 using the scaling transformation and the Floquet-Bloch decomposition we reduce the problem to the study of the generalized resolvent $\left(A(k, \varepsilon)+\varepsilon^{2} Q\right)^{-1}$ of the operator (1.12). In Section 4, the operator family $A_{1}(k)$ is studied by using method of [BSu1]; the spectral germ of $A_{1}(k)$ is introduced. In Section 5. Theorem 1 is reduced to Theorem 4, which gives approximation of the operator $\left(A(k, \varepsilon)+\varepsilon^{2} Q\right)^{-1}$ in terms of the spectral germ. Theorem 4 is proved in Sections 6 8 , this is technical part of the paper. Section 9 is devoted to application of Theorem 1 to homogenization problem for the Schrödinger operator with a singular potential periodic in one direction. Finally, in Section 10 generalizations of main results for the case of arbitrary dimension are formulated.

\subsection{Notation}

Let $\mathfrak{H}$ and $\mathfrak{G}$ be separable Hilbert spaces. The symbols $(\cdot, \cdot)_{\mathfrak{H}},\|\cdot\|_{\mathfrak{H}}$ stand for the inner product and the norm in $\mathfrak{H}$; the symbol $\|T\|_{\mathfrak{H} \rightarrow \mathfrak{G}}$ denotes the norm of a bounded operator $T: \mathfrak{H} \rightarrow \mathfrak{G}$. Sometimes we omit the indices if this does not lead to confusion. If $\mathfrak{N}$ is a subspace in $\mathfrak{H}$, then its orthogonal complement is denoted by $\mathfrak{N}^{\perp}$. If $P$ is the orthogonal projection of $\mathfrak{H}$ onto $\mathfrak{N}$, then $P^{\perp}$ denotes the orthogonal projection of $\mathfrak{H}$ onto $\mathfrak{N}^{\perp}$. in $\mathbb{C}^{d}$.

By $\mathbf{1}_{d}$ we denote the unit $(d \times d)$-matrix. The symbol $\langle\cdot, \cdot\rangle_{\mathbb{C}^{d}}$ stands for the standard inner product

By $H^{s}(\mathcal{D}), s \in \mathbb{R}$, we denote the Sobolev spaces in a domain $\mathcal{D} \subset \mathbb{R}^{d}$.

\section{Definition of the operators. Main results}

\subsection{Statement of the problem}

Let $\mathbf{x}=\left(x_{1}, x_{2}\right) \in \mathbb{R}^{2}$. We use the notation $\partial_{j}=\frac{\partial}{\partial x_{j}}, D_{j}=-i \partial_{j}, j=1,2$. Assume that $g_{j}(\mathbf{x}), j=1,2$, are real-valued measurable functions in $\mathbb{R}^{2}$ such that

$$
0<c_{0} \leq g_{j}(\mathbf{x}) \leq c_{1}<\infty, \quad j=1,2, \quad \text { a. e. } \mathbf{x} \in \mathbb{R}^{2} .
$$

The functions $g_{j}\left(x_{1}, x_{2}\right)$ are assumed to be periodic in $x_{1}$ with period 1 :

$$
g_{j}\left(x_{1}+1, x_{2}\right)=g_{j}\left(x_{1}, x_{2}\right), \quad \mathbf{x} \in \mathbb{R}^{2}, j=1,2 .
$$


Suppose also that $g_{j}\left(x_{1}, x_{2}\right)$ are Lipschitz class with respect to $x_{2}$ :

$$
\underset{\mathbf{x} \in \mathbb{R}^{2}}{\operatorname{ess} \sup }\left|\partial_{2} g_{j}(\mathbf{x})\right| \leq c_{2}<\infty, \quad j=1,2 .
$$

In the space $L_{2}\left(\mathbb{R}^{2}\right)$ we consider the operator $A_{\varepsilon}$ given formally by the differential expression (1.9). Precisely, $A_{\varepsilon}$ is defined as a selfadjoint operator in $L_{2}\left(\mathbb{R}^{2}\right)$ generated by the closed quadratic form

$$
a_{\varepsilon}[u, u]=\int_{\mathbb{R}^{2}}\left(g_{1}\left(\frac{x_{1}}{\varepsilon}, x_{2}\right)\left|D_{1} u\right|^{2}+g_{2}\left(\frac{x_{1}}{\varepsilon}, x_{2}\right)\left|D_{2} u\right|^{2}\right) d \mathbf{x}, \quad u \in H^{1}\left(\mathbb{R}^{2}\right), \varepsilon>0 .
$$

Let $Q(\mathbf{x})=Q\left(x_{1}, x_{2}\right)$ be a real-valued measurable function in $\mathbb{R}^{2}$ such that

$$
\begin{gathered}
Q\left(x_{1}+1, x_{2}\right)=Q\left(x_{1}, x_{2}\right), \quad \mathbf{x} \in \mathbb{R}^{2}, \\
0<c_{3} \leq Q(\mathbf{x}) \leq c_{4}<\infty, \quad \text { a. e. } \mathbf{x} \in \mathbb{R}^{2} .
\end{gathered}
$$

Suppose also that $Q(\mathbf{x})$ is Lipschitz class with respect to $x_{2}$ :

$$
\underset{\mathbf{x} \in \mathbb{R}^{2}}{\operatorname{ess} \sup }\left|\partial_{2} Q(\mathbf{x})\right| \leq c_{5}<+\infty .
$$

We denote

$$
Q^{\varepsilon}(\mathbf{x})=Q\left(\frac{x_{1}}{\varepsilon}, x_{2}\right) .
$$

Our goal is to study the behavior of the generalized resolvent $\left(A_{\varepsilon}+Q^{\varepsilon}\right)^{-1}$ as $\varepsilon \rightarrow 0$.

\subsection{Main result}

We find the effective operator $A^{0}$ of the same form as $A$, but with coefficients independent of $x_{1}$, and the function $Q^{0}\left(x_{2}\right)$ such that

$$
\left(A_{\varepsilon}+Q^{\varepsilon}\right)^{-1} \rightarrow\left(A^{0}+Q^{0}\right)^{-1} \text { as } \varepsilon \rightarrow 0,
$$

where convergence is understood in the operator norm in $L_{2}\left(\mathbb{R}^{2}\right)$.

The effective operator $A^{0}$ is a selfadjoint operator in $L_{2}\left(\mathbb{R}^{2}\right)$ generated by the quadratic form

$$
a^{0}[u, u]=\int_{\mathbb{R}^{2}}\left(g_{1}^{0}\left(x_{2}\right)\left|D_{1} u\right|^{2}+g_{2}^{0}\left(x_{2}\right)\left|D_{2} u\right|^{2}\right) d \mathbf{x}, \quad u \in H^{1}\left(\mathbb{R}^{2}\right) .
$$

Here

$$
\begin{gathered}
g_{1}^{0}\left(x_{2}\right)=\left(\int_{0}^{1} g_{1}\left(x_{1}, x_{2}\right)^{-1} d x_{1}\right)^{-1}, \\
g_{2}^{0}\left(x_{2}\right)=\int_{0}^{1} g_{2}\left(x_{1}, x_{2}\right) d x_{1} .
\end{gathered}
$$

Note that conditions (2.1), (2.3) imply the following inequalities for the effective coefficients (2.10), (2.11):

$$
\begin{gathered}
0<c_{0} \leq g_{j}^{0}\left(x_{2}\right) \leq c_{1}<+\infty, \quad x_{2} \in \mathbb{R}, \quad j=1,2, \\
\quad \underset{x_{2} \in \mathbb{R}}{\operatorname{ess} \sup _{2}}\left|\partial_{2} g_{1}^{0}\left(x_{2}\right)\right| \leq c_{2}\left(\frac{c_{1}}{c_{0}}\right)^{2}, \\
\quad \underset{x_{2} \in \mathbb{R}}{\operatorname{ess} \sup _{2}}\left|\partial_{2} g_{2}^{0}\left(x_{2}\right)\right| \leq c_{2} .
\end{gathered}
$$

Due to conditions (2.13), (2.14), $A^{0}$ can be given by the differential expression $A^{0}=g_{1}^{0}\left(x_{2}\right) D_{1}^{2}+$ $D_{2} g_{2}^{0}\left(x_{2}\right) D_{2}$ on the domain $H^{2}\left(\mathbb{R}^{2}\right)$. 
Next, let $Q^{0}\left(x_{2}\right)$ be the mean value of $Q\left(x_{1}, x_{2}\right)$ :

$$
Q^{0}\left(x_{2}\right)=\int_{0}^{1} Q\left(x_{1}, x_{2}\right) d x_{1} .
$$

From (2.6) and (2.7) it follows that

$$
\begin{gathered}
0<c_{3} \leq Q^{0}\left(x_{2}\right) \leq c_{4}<+\infty, \quad x_{2} \in \mathbb{R}, \\
\underset{x_{2} \in \mathbb{R}}{\operatorname{ess} \sup }\left|\partial_{2} Q^{0}\left(x_{2}\right)\right| \leq c_{5}<+\infty .
\end{gathered}
$$

The main result is given in the following theorem.

Theorem 1 Suppose that conditions (2.1)-(2.3) are satisfied and $A_{\varepsilon}$ is the operator in $L_{2}\left(\mathbb{R}^{2}\right)$ that corresponds to the form (2.4). Let $A^{0}$ be the operator corresponding to the form (2.9), where the coefficients are defined by (2.10), (2.11). Let $Q(\mathbf{x})$ be a function in $\mathbb{R}^{2}$ satisfying (2.5)-(2.7), and $Q^{\varepsilon}$ is defined by (2.8). Let $Q^{0}$ be defined by (2.15). Then we have

$$
\left\|\left(A_{\varepsilon}+Q^{\varepsilon}\right)^{-1}-\left(A^{0}+Q^{0}\right)^{-1}\right\|_{L_{2}\left(\mathbb{R}^{2}\right) \rightarrow L_{2}\left(\mathbb{R}^{2}\right)} \leq C \varepsilon, \quad 0<\varepsilon \leq 1 .
$$

The constant $C$ depends only on $c_{j}, j=0, \ldots, 5$.

\section{Reduction to operators in the strip $\Omega$}

\subsection{Scaling transformation}

We denote by $T_{\varepsilon}, \varepsilon>0$, the unitary scaling transformation in $L_{2}\left(\mathbb{R}^{2}\right)$ defined by the formula

$$
\left(T_{\varepsilon} u\right)\left(x_{1}, x_{2}\right)=\varepsilon^{\frac{1}{2}} u\left(\varepsilon x_{1}, x_{2}\right), \quad\left(x_{1}, x_{2}\right) \in \mathbb{R}^{2} .
$$

Let $A(\varepsilon)$ be the operator in $L_{2}\left(\mathbb{R}^{2}\right)$ generated by the quadratic form

$$
a(\varepsilon)[u, u]=\int_{\mathbb{R}^{2}}\left(g_{1}(\mathbf{x})\left|D_{1} u\right|^{2}+\varepsilon^{2} g_{2}(\mathbf{x})\left|D_{2} u\right|^{2}\right) d \mathbf{x}, \quad u \in H^{1}\left(\mathbb{R}^{2}\right) .
$$

We have the obvious identity

$$
A_{\varepsilon}=\varepsilon^{-2} T_{\varepsilon}^{*} A(\varepsilon) T_{\varepsilon} .
$$

Clearly, for the operator $\left[Q^{\varepsilon}\right]$ of multiplication by the function $Q^{\varepsilon}(\mathbf{x})$, we have

$$
\left[Q^{\varepsilon}\right]=T_{\varepsilon}^{*}[Q] T_{\varepsilon} .
$$

From (3.2) and (3.3) it follows that

$$
\left(A_{\varepsilon}+Q^{\varepsilon}\right)^{-1}=\varepsilon^{2} T_{\varepsilon}^{*}\left(A(\varepsilon)+\varepsilon^{2} Q\right)^{-1} T_{\varepsilon} .
$$

A similar representation is true for $\left(A^{0}+Q^{0}\right)^{-1}$ :

$$
\left(A^{0}+Q^{0}\right)^{-1}=\varepsilon^{2} T_{\varepsilon}^{*}\left(A^{0}(\varepsilon)+\varepsilon^{2} Q^{0}\right)^{-1} T_{\varepsilon},
$$

where $A^{0}(\varepsilon)$ corresponds to the quadratic form

$$
a^{0}(\varepsilon)[u, u]=\int_{\mathbb{R}^{2}}\left(g_{1}^{0}\left(x_{2}\right)\left|D_{1} u\right|^{2}+\varepsilon^{2} g_{2}^{0}\left(x_{2}\right)\left|D_{2} u\right|^{2}\right) d \mathbf{x}, \quad u \in H^{1}\left(\mathbb{R}^{2}\right) .
$$

We can also define $A^{0}(\varepsilon)$ by the differential expression $g_{1}^{0}\left(x_{2}\right) D_{1}^{2}+\varepsilon^{2} D_{2} g_{2}^{0}\left(x_{2}\right) D_{2}$ on domain $H^{2}\left(\mathbb{R}^{2}\right)$.

Using (3.4), (3.5) and the fact that $T_{\varepsilon}$ is unitary operator in $L_{2}\left(\mathbb{R}^{2}\right)$, we reduce Theorem 1 to the following theorem. 
Theorem 2 Suppose that conditions (2.1)-(2.3) are satisfied. Let $A(\varepsilon)$ be the operator in $L_{2}\left(\mathbb{R}^{2}\right)$ that corresponds to the quadratic form (3.1). Let $A^{0}(\varepsilon)$ be the operator corresponding to the form (3.6) with coefficients defined by (2.10), (2.11). Let $Q(\mathbf{x})$ be a function in $\mathbb{R}^{2}$ satisfying (2.5)-(2.7), and let $Q^{0}\left(x_{2}\right)$ be defined by (2.15). Then we have

$$
\left\|\left(A(\varepsilon)+\varepsilon^{2} Q\right)^{-1}-\left(A^{0}(\varepsilon)+\varepsilon^{2} Q^{0}\right)^{-1}\right\|_{L_{2}\left(\mathbb{R}^{2}\right) \rightarrow L_{2}\left(\mathbb{R}^{2}\right)} \leq C \varepsilon^{-1}, \quad 0<\varepsilon \leq 1 .
$$

The constant $C$ depends only on $c_{j}, j=0, \ldots, 5$.

\subsection{Direct integral decomposition for $A(\varepsilon)$}

First, we define the Gelfand transformation $\mathcal{U}$. We denote

$$
\Omega=(0,1) \times \mathbb{R},
$$

and consider the Hilbert space

$$
\mathcal{K}=\int_{[-\pi, \pi)} \oplus L_{2}(\Omega) d k=L_{2}\left([-\pi, \pi) ; L_{2}(\Omega)\right)=L_{2}([-\pi, \pi) \times \Omega),
$$

which is the direct integral with constant fibers. Initially, the operator $\mathcal{U}: L_{2}\left(\mathbb{R}^{2}\right) \rightarrow \mathcal{K}$ is defined on the functions $f \in C_{0}^{\infty}\left(\mathbb{R}^{2}\right)$ by the formula

$$
(\mathcal{U} f)(\mathbf{x}, k)=(2 \pi)^{-1 / 2} \sum_{m \in \mathbb{Z}} e^{-i k\left(x_{1}+m\right)} f\left(x_{1}+m, x_{2}\right), \quad \mathbf{x}=\left(x_{1}, x_{2}\right) \in \Omega, k \in[-\pi, \pi) .
$$

Next, $\mathcal{U}$ extends by continuity to a unitary mapping of $L_{2}\left(\mathbb{R}^{2}\right)$ onto $\mathcal{K}$.

In $L_{2}(\Omega)$, we consider the operator $A(k, \varepsilon)$ generated by the quadratic form

$$
a(k, \varepsilon)[u, u]=\int_{\Omega}\left(g_{1}(\mathbf{x})\left|\left(D_{1}+k\right) u\right|^{2}+\varepsilon^{2} g_{2}(\mathbf{x})\left|D_{2} u\right|^{2}\right) d \mathbf{x}, \quad u \in \widetilde{H}^{1}(\Omega), k \in[-\pi, \pi), \varepsilon>0 .
$$

Here by $\widetilde{H}^{s}(\Omega)$ we denote the subspace of $H^{s}(\Omega)$ formed by the functions whose 1-periodic extension (in $x_{1}$ ) to $\mathbb{R}^{2}$ belongs to $H_{\text {loc }}^{s}\left(\mathbb{R}^{2}\right)$.

The operator $A(\varepsilon)$ is decomposed in the direct integral of operators $A(k, \varepsilon)$ with the help of the Gelfand transformation $\mathcal{U}$ :

$$
\mathcal{U} A(\varepsilon) \mathcal{U}^{-1}=\int_{[-\pi, \pi)} \oplus A(k, \varepsilon) d k
$$

Then, for the generalized resolvent $\left(A(\varepsilon)+\varepsilon^{2} Q\right)^{-1}$ we have

$$
\mathcal{U}\left(A(\varepsilon)+\varepsilon^{2} Q\right)^{-1} \mathcal{U}^{-1}=\int_{[-\pi, \pi)} \oplus\left(A(k, \varepsilon)+\varepsilon^{2} Q\right)^{-1} d k .
$$

Let $A^{0}(k, \varepsilon)$ be the operator in $L_{2}(\Omega)$ corresponding to the quadratic form

$$
a^{0}(k, \varepsilon)[u, u]=\int_{\Omega}\left(g_{1}^{0}\left(x_{2}\right)\left|\left(D_{1}+k\right) u\right|^{2}+\varepsilon^{2} g_{2}^{0}\left(x_{2}\right)\left|D_{2} u\right|^{2}\right) d \mathbf{x}, \quad u \in \widetilde{H}^{1}(\Omega), k \in[-\pi, \pi), \varepsilon>0 .
$$

We can also define $A^{0}(k, \varepsilon)$ by the differential expression $g_{1}^{0}\left(x_{2}\right)\left(D_{1}+k\right)^{2}+\varepsilon^{2} D_{2} g_{2}^{0}\left(x_{2}\right) D_{2}$ on the domain $\widetilde{H}^{2}(\Omega)$. For $A^{0}(\varepsilon)$ we also have the direct integral decomposition:

$$
\mathcal{U} A^{0}(\varepsilon) \mathcal{U}^{-1}=\int_{[-\pi, \pi)} \oplus A^{0}(k, \varepsilon) d k .
$$


Then, for the generalized resolvent $\left(A^{0}(\varepsilon)+\varepsilon^{2} Q^{0}\right)^{-1}$ we have

$$
\mathcal{U}\left(A^{0}(\varepsilon)+\varepsilon^{2} Q^{0}\right)^{-1} \mathcal{U}^{-1}=\int_{[-\pi, \pi)} \oplus\left(A^{0}(k, \varepsilon)+\varepsilon^{2} Q^{0}\right)^{-1} d k .
$$

Using (3.12), (3.15) and the fact that $\mathcal{U}$ is unitary transformation, we see that Theorem 2 (and then also Theorem (1) is a direct consequence of the following statement.

Theorem 3 Suppose that conditions (2.1)-(2.3) are satisfied. Let $A(k, \varepsilon)$ be the operator in $L_{2}(\Omega)$ generated by the quadratic form (3.11). Let $A^{0}(k, \varepsilon)$ be the operator in $L_{2}(\Omega)$ corresponding to the form (3.13), where the coefficients are defined by (2.10), (2.11). Let $Q(\mathbf{x})$ be a function satisfying (2.5)-(2.7), and let $Q^{0}\left(x_{2}\right)$ be defined by (2.15). Then we have

$$
\left\|\left(A(k, \varepsilon)+\varepsilon^{2} Q\right)^{-1}-\left(A^{0}(k, \varepsilon)+\varepsilon^{2} Q^{0}\right)^{-1}\right\|_{L_{2}(\Omega) \rightarrow L_{2}(\Omega)} \leq C \varepsilon^{-1}, \quad 0<\varepsilon \leq 1, k \in[-\pi, \pi) .
$$

The constant $C$ depends only on $c_{j}, j=0, \ldots, 5$.

\section{The operator pencil $A_{1}(k)$}

\subsection{Definition of $A_{1}(k)$. Direct integral decomposition}

If we formally put $\varepsilon=0$, then the operator $A(k, \varepsilon)$ defined in Subsection 3.2 turns into the operator $A_{1}(k)$ formally given by the expression $\left(D_{1}+k\right) g_{1}(\mathbf{x})\left(D_{1}+k\right)$ with periodic boundary conditions on $\partial \Omega$. Precisely, $A_{1}(k)$ is the selfadjoint operator in $L_{2}(\Omega)$ generated by the quadratic form

$$
\begin{gathered}
a_{1}(k)[u, u]=\int_{\Omega} g_{1}(\mathbf{x})\left|\left(D_{1}+k\right) u\right|^{2} d \mathbf{x}, \\
\operatorname{Dom} a_{1}(k)=L_{2}\left(\mathbb{R} ; \widetilde{H}^{1}(0,1)\right)=\int_{\mathbb{R}} \oplus \widetilde{H}^{1}(0,1) d x_{2} .
\end{gathered}
$$

Here $\widetilde{H}^{1}(0,1)$ is the subspace of $H^{1}(0,1)$ formed by the functions whose 1-periodic extension to $\mathbb{R}$ belongs to $H_{\text {loc }}^{1}(\mathbb{R})$.

In this section we study the operator family $A_{1}(k)$. For this, it suffices to impose only condition (2.1) with $j=1$. Now it is convenient to interpret the space $L_{2}(\Omega)$ as a direct integral in $x_{2}$ with constant fibers $L_{2}(0,1)$ :

$$
L_{2}(\Omega)=\int_{\mathbb{R}} \oplus L_{2}(0,1) d x_{2} .
$$

The operator $A_{1}(k)$ acts in the space (4.3) "layerwise", i. e., it is the operator of multiplication by the operator-valued function $A_{1}\left(k ; x_{2}\right)$ :

$$
A_{1}(k)=\int_{\mathbb{R}} \oplus A_{1}\left(k ; x_{2}\right) d x_{2},
$$

where $A_{1}\left(k, x_{2}\right)$ is the selfadjoint operator in $L_{2}(0,1)$ generated by the form

$$
a_{1}\left(k ; x_{2}\right)[u, u]=\int_{0}^{1} g_{1}\left(x_{1}, x_{2}\right)\left|\left(D_{1}+k\right) u\right|^{2} d x_{1}, \quad u \in \widetilde{H}^{1}(0,1), x_{2} \in \mathbb{R}, k \in[-\pi, \pi) .
$$




\subsection{The operator family $A_{1}\left(k ; x_{2}\right)$}

The operator family $A_{1}\left(k ; x_{2}\right)$ can be studied by the method suggested in BSu1. This family admits a factorization

$$
\begin{gathered}
A_{1}\left(k ; x_{2}\right)=\left(X\left(k ; x_{2}\right)\right)^{*} X\left(k ; x_{2}\right), \\
X\left(k ; x_{2}\right)=X_{0}\left(x_{2}\right)+k X_{1}\left(x_{2}\right),
\end{gathered}
$$

where $X_{0}\left(x_{2}\right)$ is the (closed) operator in $L_{2}(0,1)$ given by the differential expression $\left(g_{1}\left(x_{1}, x_{2}\right)\right)^{\frac{1}{2}} D_{1}$ on the domain $\widetilde{H}^{1}(0,1)$, and $X_{1}\left(x_{2}\right)$ is the bounded operator in $L_{2}(0,1)$ of multiplication by the function $\left(g_{1}\left(x_{1}, x_{2}\right)\right)^{\frac{1}{2}}$. Then the operator (4.7) is closed on the domain $\widetilde{H}^{1}(0,1)$. Obviously, the kernel

$$
\widetilde{\mathfrak{N}}=\operatorname{Ker} A_{1}\left(0 ; x_{2}\right)=\operatorname{Ker} X_{0}\left(x_{2}\right)=\left\{u \in L_{2}(0,1): u=\text { const }\right\}
$$

is one-dimensional. Let $d_{0}\left(x_{2}\right)$ be the distance from the point $\lambda_{0}=0$ to the rest of the spectrum of $A_{1}\left(0 ; x_{2}\right)$. Let us estimate $d_{0}\left(x_{2}\right)$ from below.

By (2.1) (with $j=1$ ), the form (4.5) with $k=0$ satisfies the estimate

$$
a_{1}\left(0 ; x_{2}\right)[u, u] \geq c_{0} \int_{0}^{1}\left|D_{1} u\left(x_{1}\right)\right|^{2} d x_{1}, \quad u \in \widetilde{H}^{1}(0,1) .
$$

Using the Fourier series expansion for $u \in \widetilde{H}^{1}(0,1)$

$$
u\left(x_{1}\right)=\sum_{m \in \mathbb{Z}} \widehat{u}_{m} e^{2 \pi i m x_{1}},
$$

and (4.9), we obtain

$$
a_{1}\left(0 ; x_{2}\right)[u, u] \geq c_{0} \sum_{m \in \mathbb{Z}} 4 \pi^{2} m^{2}\left|\widehat{u}_{m}\right|^{2}, \quad u \in \widetilde{H}^{1}(0,1) .
$$

If $u$ is orthogonal to $\widetilde{\mathfrak{N}}$ in $L_{2}(0,1)$, then $\widehat{u}_{0}=0$, and from (4.10) we deduce that

$$
a_{1}\left(0 ; x_{2}\right)[u, u] \geq 4 \pi^{2} c_{0}\|u\|_{L_{2}(0,1)}^{2}, \quad u \in \widetilde{H}^{1}(0,1), u \perp \widetilde{\mathfrak{N}} .
$$

It follows that

$$
d_{0}\left(x_{2}\right) \geq 4 \pi^{2} c_{0}=: d_{0}, \quad x_{2} \in \mathbb{R} .
$$

In accordance with [BSu1, Ch.1, $\S 1$, we choose the positive number $\delta$ such that $8 \delta<d_{0}$. We can put

$$
\delta=\frac{\pi^{2} c_{0}}{4}
$$

Next, by the upper estimate (2.1), we have

$$
\left\|X_{1}\left(x_{2}\right)\right\|_{L_{2}(0,1) \rightarrow L_{2}(0,1)} \leq\left\|g_{1}\left(\cdot, x_{2}\right)\right\|_{L_{\infty}(0,1)}^{\frac{1}{2}} \leq c_{1}^{\frac{1}{2}}, \quad x_{2} \in \mathbb{R} .
$$

As in BSu1, Ch. 1, $\S 1$ ], we choose a number $t_{0}$ such that $t_{0} \leq \delta^{\frac{1}{2}}\left\|X_{1}\left(x_{2}\right)\right\|^{-1}$ for all $x_{2} \in \mathbb{R}$. We put

$$
t_{0}=\frac{\pi}{2} c_{0}^{\frac{1}{2}} c_{1}^{-\frac{1}{2}}
$$

Let $F\left(k ; x_{2} ; \sigma\right)$ be the spectral projection of the operator $A_{1}\left(k ; x_{2}\right)$ corresponding to the interval $[0, \sigma]$. From Proposition 1.2 of [BSu1, Ch. 1] it follows that

$$
\operatorname{dim} F\left(k ; x_{2} ; \delta\right) L_{2}(0,1)=\operatorname{dim} F\left(k ; x_{2} ; 3 \delta\right) L_{2}(0,1)=1, \quad|k|=t \leq t_{0} .
$$


It means that for $|k| \leq t_{0}$ and all $x_{2} \in \mathbb{R}$ the operator $A_{1}\left(k ; x_{2}\right)$ has only one eigenvalue lying in the interval $[0, \delta]$, while the interval $(\delta, 3 \delta)$ is free of its spectrum. We put

$$
F\left(k ; x_{2}\right)=F\left(k ; x_{2} ; \delta\right) .
$$

Let $\widetilde{P}$ be the orthogonal projection of $L_{2}(0,1)$ onto the subspace $\widetilde{\mathfrak{N}}$. By Theorem 4.1 of [BSu1, Ch.1], we have the following approximation for the operator (4.17):

$$
\begin{aligned}
& F\left(k ; x_{2}\right)=\widetilde{P}+t \Phi\left(k ; x_{2}\right), \quad t=|k| \leq t_{0}, x_{2} \in \mathbb{R}, \\
& \left\|\Phi\left(k ; x_{2}\right)\right\|_{L_{2}(0,1) \rightarrow L_{2}(0,1)} \leq C_{1}, \quad t \leq t_{0}, x_{2} \in \mathbb{R},
\end{aligned}
$$

where

$$
C_{1}=\beta_{1} \delta^{-\frac{1}{2}} \sup _{x_{2} \in \mathbb{R}}\left\|X_{1}\left(x_{2}\right)\right\| \leq 2 \beta_{1} c_{1}^{\frac{1}{2}} c_{0}^{-\frac{1}{2}} \pi^{-1},
$$

and $\beta_{1}$ is an absolute constant.

In accordance with [BSu1, we introduce the spectral germ $S\left(x_{2}\right)$ of the operator family $A_{1}\left(k ; x_{2}\right)$. The germ is a selfadjoint operator acting in the space $\widetilde{\mathfrak{N}}$. In our case (i. e., for the operator of the form $-\frac{d}{d x} g(x) \frac{d}{d x}$ ), the germ is calculated explicitly (see [BSu1, Ch.5, $\left.\S 1\right]$ ). The operator $S\left(x_{2}\right)$ is multiplication by the number $g_{1}^{0}\left(x_{2}\right)$, defined by (2.10).

By Theorem 4.3 of [BSu1, Ch.1], we have the following approximation for the operator $A_{1}\left(k ; x_{2}\right) F\left(k ; x_{2}\right)$ :

$$
\begin{gathered}
A_{1}\left(k ; x_{2}\right) F\left(k ; x_{2}\right)=t^{2} S\left(x_{2}\right) \widetilde{P}+t^{3} \Psi\left(k ; x_{2}\right), \quad t=|k| \leq t_{0}, x_{2} \in \mathbb{R}, \\
\left\|\Psi\left(k ; x_{2}\right)\right\|_{L_{2}(0,1) \rightarrow L_{2}(0,1)} \leq C_{2}, \quad t \leq t_{0}, x_{2} \in \mathbb{R},
\end{gathered}
$$

where

$$
C_{2}=\beta_{2} \delta^{-\frac{1}{2}} \sup _{x_{2} \in \mathbb{R}}\left\|X_{1}\left(x_{2}\right)\right\|^{3} \leq 2 \beta_{2} \pi^{-1} c_{1}^{\frac{3}{2}} c_{0}^{-\frac{1}{2}},
$$

and $\beta_{2}$ is an absolute constant.

\subsection{The spectral germ of the operator family $A_{1}(k)$}

We return to the family (4.4) acting in $L_{2}(\Omega)$. From (4.4) and (4.16) it follows that the interval $(\delta, 3 \delta)$ is free of the spectrum of $A_{1}(k)$, if $|k| \leq t_{0}$. By (4.4) and (4.6), we have $A_{1}(k)=X(k)^{*} X(k)$, where $X(k)=$ $\int_{\mathbb{R}} \oplus X\left(k ; x_{2}\right) d x_{2}$. Then, by (4.7), $X(k)=X_{0}+k X_{1}, X_{0}=\int_{\mathbb{R}} \oplus X_{0}\left(x_{2}\right) d x_{2}, X_{1}=\int_{\mathbb{R}} \oplus X_{1}\left(x_{2}\right) d x_{2}$.

Clearly, $X_{0}$ is the operator in $L_{2}(\Omega)$ given by $\left(g_{1}(x)\right)^{\frac{1}{2}} D_{1}$ on the domain (4.2), and $X_{1}$ is the bounded operator in $L_{2}(\Omega)$ of multiplication by the function $\left(g_{1}(x)\right)^{\frac{1}{2}}$.

For the operator $A_{1}(0)$ with $k=0$ we have

$$
\mathfrak{N}=\operatorname{Ker} A_{1}(0)=\operatorname{Ker} X_{0}=\left\{u \in L_{2}(\Omega): u=u\left(x_{2}\right)\right\} .
$$

Thus, the kernel $\mathfrak{N}$ consists of functions in $L_{2}(\Omega)$ depending only on the second variable $x_{2}$. It can be identified with $L_{2}(\mathbb{R})$. Obviously, we can write

$$
\mathfrak{N}=\int_{\mathbb{R}} \oplus \widetilde{\mathfrak{N}} d x_{2} .
$$

Also, the orthogonal projection $P$ of $L_{2}(\Omega)$ onto $\mathfrak{N}$ can be represented as the direct integral

$$
P=\int_{\mathbb{R}} \oplus \widetilde{P} d x_{2} .
$$

Now, we consider the operator $S$ in $\mathfrak{N}$ which is the direct integral of $S\left(x_{2}\right)$ :

$$
S \stackrel{\text { def }}{=} \int_{\mathbb{R}} \oplus S\left(x_{2}\right) d x_{2} .
$$


Then $S$ is the operator in $\mathfrak{N} \equiv L_{2}(\mathbb{R})$ of multiplication by the function $g_{1}^{0}\left(x_{2}\right)$. The operator $S$ is called the spectral germ of $A_{1}(k)$.

Let $F(k)$ denote the spectral projection of the operator $A_{1}(k)$ corresponding to the interval $[0, \delta]$. Since $A_{1}(k)$ is represented as the direct integral (4.4), we have

$$
F(k)=\int_{\mathbb{R}} \oplus F\left(k ; x_{2}\right) d x_{2} .
$$

From (4.18), (4.26) and (4.28) it follows that

$$
\begin{gathered}
F(k)=P+t \Phi(k), \quad t=|k| \leq t_{0}, \\
\Phi(k)=\int_{\mathbb{R}} \oplus \Phi\left(k ; x_{2}\right) d x_{2} .
\end{gathered}
$$

Similarly, relations (4.4), (4.21), (4.26)-(4.28) imply that

$$
\begin{gathered}
A_{1}(k) F(k)=t^{2} S P+t^{3} \Psi(k), \quad t=|k| \leq t_{0}, \\
\Psi(k)=\int_{\mathbb{R}} \oplus \Psi\left(k ; x_{2}\right) d x_{2} .
\end{gathered}
$$

By (4.19) and (4.22), the operators (4.30) and (4.32) satisfy the estimates

$$
\begin{aligned}
& \|\Phi(k)\|_{L_{2}(\Omega) \rightarrow L_{2}(\Omega)} \leq C_{1}, \quad t=|k| \leq t_{0}, \\
& \|\Psi(k)\|_{L_{2}(\Omega) \rightarrow L_{2}(\Omega)} \leq C_{2}, \quad t=|k| \leq t_{0} .
\end{aligned}
$$

\section{Approximation for the generalized resolvent of $A(k, \varepsilon)$}

\subsection{Approximation in terms of the operator $S(k, \varepsilon)$}

Now we return to the operator family $A(k, \varepsilon)$ introduced in Subsection 3.2 Our goal is to approximate the generalized resolvent $\left(A(k, \varepsilon)+\varepsilon^{2} Q\right)^{-1}$.

In the space $\mathfrak{N} \equiv L_{2}(\mathbb{R})$ (see 4.24$)$ ), we consider the quadratic form

$$
\begin{gathered}
s(k, \varepsilon)[\omega, \omega]=\int_{\mathbb{R}}\left(g_{1}^{0}\left(x_{2}\right) k^{2}\left|\omega\left(x_{2}\right)\right|^{2}+\varepsilon^{2} g_{2}^{0}\left(x_{2}\right)\left|D_{2} \omega\left(x_{2}\right)\right|^{2}\right) d x_{2}, \quad k \in[-\pi, \pi), \varepsilon>0, \\
\operatorname{Dom} s(k, \varepsilon)=H^{1}(\mathbb{R}) .
\end{gathered}
$$

The selfadjoint operator in $L_{2}(\mathbb{R})$ corresponding to this form is denoted by $S(k, \varepsilon)$. Clearly, under conditions (2.12), (2.14), the operator $S(k, \varepsilon)$ is given by the differential expression

$$
S(k, \varepsilon)=g_{1}^{0}\left(x_{2}\right) k^{2}+\varepsilon^{2} D_{2} g_{2}^{0}\left(x_{2}\right) D_{2},
$$

on the domain

$$
\operatorname{Dom} S(k, \varepsilon)=H^{2}(\mathbb{R})
$$

Note that the first summand in (5.3) corresponds to $t^{2} S$.

The key role in the proof of Theorem 3 is played by the following theorem, which gives an approximation for the generalized resolvent of $A(k, \varepsilon)$ in terms of the operator $S(k, \varepsilon)$.

Theorem 4 Suppose that conditions (2.1)-(2.3) are satisfied. Let $A(k, \varepsilon)$ be the operator in $L_{2}(\Omega)$ generated by the quadratic form (3.11). Let $S(k, \varepsilon)$ be the operator in $\mathfrak{N}$ defined by (5.3), (5.4), where the coefficients are defined by (2.10), (2.11). Let $Q(\mathbf{x})$ be a function satisfying (2.5)-(2.7) and let $Q^{0}\left(x_{2}\right)$ be defined by (2.15). Let $P$ be the orthogonal projection of $L_{2}(\Omega)$ onto the subspace [4.24). Then we have

$$
\left\|\left(A(k, \varepsilon)+\varepsilon^{2} Q\right)^{-1}-\left(S(k, \varepsilon)+\varepsilon^{2} Q^{0}\right)^{-1} P\right\|_{L_{2}(\Omega) \rightarrow L_{2}(\Omega)} \leq \widetilde{C} \varepsilon^{-1}, \quad|k| \leq t_{0}, \quad 0<\varepsilon \leq 1 .
$$

The number $t_{0}$ is defined by 4.15 . The constant $\widetilde{C}$ depends only on $c_{j}, j=0, \ldots, 5$. 


\subsection{Deduction of Theorem 3 from Theorem 4}

Let $A^{0}(k, \varepsilon)$ be the operator in $L_{2}(\Omega)$ corresponding to the form (3.13). For $A^{0}(k, \varepsilon)$, we define the operator $S^{0}(k, \varepsilon)$ by the same rule as for $A(k, \varepsilon)$. Clearly, $S^{0}(k, \varepsilon)=S(k, \varepsilon)$. Applying Theorem 4 to the operator $\left(A^{0}(k, \varepsilon)+\varepsilon^{2} Q^{0}\right)^{-1}$, we obtain

$$
\left\|\left(A^{0}(k, \varepsilon)+\varepsilon^{2} Q^{0}\right)^{-1}-\left(S(k, \varepsilon)+\varepsilon^{2} Q^{0}\right)^{-1} P\right\|_{L_{2}(\Omega) \rightarrow L_{2}(\Omega)} \leq \widetilde{C}^{\prime} \varepsilon^{-1}, \quad|k| \leq t_{0}, 0<\varepsilon \leq 1 .
$$

Since the coefficients $g_{j}^{0}\left(x_{2}\right), j=1,2$, satisfy estimates (2.12) with the same constants $c_{0}$ and $c_{1}$ as in (2.1), the number $t_{0}$ in (5.5) and (5.6) is one and the same (see (4.15)). Also, using the statement of Theorem 4 concerning the constant $\widetilde{C}$ and (2.13), (2.14), we see that $\widetilde{C}^{\prime}$ is controlled in terms of the constants $c_{j}, j=0, \ldots, 5$.

Combining (5.5) and (5.6), we have

$$
\left\|\left(A(k, \varepsilon)+\varepsilon^{2} Q\right)^{-1}-\left(A^{0}(k, \varepsilon)+\varepsilon^{2} Q^{0}\right)^{-1}\right\|_{L_{2}(\Omega) \rightarrow L_{2}(\Omega)} \leq\left(\widetilde{C}+\widetilde{C}^{\prime}\right) \varepsilon^{-1}, \quad|k| \leq t_{0}, \quad 0<\varepsilon \leq 1 .
$$

So, we have obtained the required estimate (3.16) for $|k| \leq t_{0}$.

If $k \in[-\pi, \pi)$ and $|k|>t_{0}$, the estimates are trivial: both terms in (3.16) are estimated by constants. Indeed, by (2.1) and (3.11),

$$
a(k, \varepsilon)[u, u] \geq c_{0} \int_{\Omega}\left(\left|\left(D_{1}+k\right) u\right|^{2}+\varepsilon^{2}\left|D_{2} u\right|^{2}\right) d \mathbf{x}, \quad u \in \widetilde{H}^{1}(\Omega), k \in[-\pi, \pi), \varepsilon>0 .
$$

Using the Fourier series expansion (in variable $x_{1}$ ) for $u \in \widetilde{H}^{1}(\Omega)$ :

$$
u\left(x_{1}, x_{2}\right)=\sum_{m \in \mathbb{Z}} \widehat{u}_{m}\left(x_{2}\right) e^{2 \pi i m x_{1}}
$$

we obtain

$$
a(k, \varepsilon)[u, u] \geq c_{0} \sum_{m \in \mathbb{Z}}(2 \pi m+k)^{2} \int_{\mathbb{R}}\left|\widehat{u}_{m}\left(x_{2}\right)\right|^{2} d x_{2}, \quad u \in \widetilde{H}^{1}(\Omega), k \in[-\pi, \pi), \varepsilon>0 .
$$

If $k \in\left[-\pi,-t_{0}\right) \cup\left(t_{0}, \pi\right)$, then $|2 \pi m+k| \geq t_{0}, m \in \mathbb{Z}$. Then (5.8) implies that

$$
a(k, \varepsilon)[u, u] \geq c_{0} t_{0}^{2}\|u\|_{L_{2}(\Omega)}^{2}, \quad u \in \widetilde{H}^{1}(\Omega), k \in\left[-\pi,-t_{0}\right) \cup\left(t_{0}, \pi\right), \varepsilon>0 .
$$

Consequently,

$$
A(k, \varepsilon) \geq c_{0} t_{0}^{2} I, \quad k \in\left[-\pi,-t_{0}\right) \cup\left(t_{0}, \pi\right), \varepsilon>0,
$$

and

$$
\left\|\left(A(k, \varepsilon)+\varepsilon^{2} Q\right)^{-1}\right\|_{L_{2}(\Omega) \rightarrow L_{2}(\Omega)} \leq c_{0}^{-1} t_{0}^{-2}, \quad k \in\left[-\pi,-t_{0}\right) \cup\left(t_{0}, \pi\right), \varepsilon>0 .
$$

The same estimate is true for $\left(A^{0}(k, \varepsilon)+\varepsilon^{2} Q^{0}\right)^{-1}$. Hence,

$$
\left\|\left(A(k, \varepsilon)+\varepsilon^{2} Q\right)^{-1}-\left(A^{0}(k, \varepsilon)+\varepsilon^{2} Q^{0}\right)^{-1}\right\|_{L_{2}(\Omega) \rightarrow L_{2}(\Omega)} \leq 2 c_{0}^{-1} t_{0}^{-2}, \quad k \in\left[-\pi,-t_{0}\right) \cup\left(t_{0}, \pi\right), \varepsilon>0 .
$$

From (5.7) and (5.12) it follows that (3.16) is valid with the constant $C=\max \left\{\widetilde{C}+\widetilde{C}^{\prime}, 2 c_{0}^{-1} t_{0}^{-2}\right\}$.

Theorem 4 will be proved in Sections 6, 7 , The proof needs some technical preparations.

\section{$6 \quad$ Preliminary estimates}

\subsection{Estimates for generalized resolvents of the operators $A(k, \varepsilon), A_{1}(k)$ and $S(k, \varepsilon)$.}

Lemma 5 We have

$$
\left\|\left(A(k, \varepsilon)+\varepsilon^{2} Q\right)^{-1}\right\|_{L_{2}(\Omega) \rightarrow L_{2}(\Omega)} \leq\left(c_{0} k^{2}+c_{3} \varepsilon^{2}\right)^{-1}, \quad k \in[-\pi, \pi), \quad \varepsilon>0,
$$




$$
\begin{gathered}
\left\|\left(A_{1}(k)+\varepsilon^{2} Q\right)^{-1}\right\|_{L_{2}(\Omega) \rightarrow L_{2}(\Omega)} \leq\left(c_{0} k^{2}+c_{3} \varepsilon^{2}\right)^{-1}, \quad k \in[-\pi, \pi), \quad \varepsilon>0, \\
\left\|\left(S(k, \varepsilon)+\varepsilon^{2} Q^{0}\right)^{-1} P\right\|_{L_{2}(\Omega) \rightarrow L_{2}(\Omega)} \leq\left(c_{0} k^{2}+c_{3} \varepsilon^{2}\right)^{-1}, \quad k \in[-\pi, \pi), \quad \varepsilon>0 .
\end{gathered}
$$

Proof. From (5.8) it follows that

$$
a(k, \varepsilon)[u, u] \geq c_{0} k^{2}\|u\|_{L_{2}(\Omega)}^{2}, \quad u \in \widetilde{H}^{1}(\Omega), k \in[-\pi, \pi), \varepsilon>0 .
$$

Combining this with the lower estimate (2.6), we see that

$$
a(k, \varepsilon)[u, u]+\varepsilon^{2} \int_{\Omega} Q(\mathbf{x})|u|^{2} d \mathbf{x} \geq\left(c_{0} k^{2}+c_{3} \varepsilon^{2}\right)\|u\|_{L_{2}(\Omega)}^{2}, \quad u \in \widetilde{H}^{1}(\Omega), k \in[-\pi, \pi), \varepsilon>0,
$$

which implies 6.1).

From (2.1) and (4.1) it follows that

$$
a_{1}(k)[u, u] \geq c_{0} \int_{\Omega}\left|\left(D_{1}+k\right) u\right|^{2} d \mathbf{x}, \quad u \in \widetilde{H}^{1}(\Omega), k \in[-\pi, \pi), \varepsilon>0 .
$$

Hence, similarly to (5.8),

$$
a_{1}(k)[u, u] \geq c_{0} k^{2}\|u\|_{L_{2}(\Omega)}^{2}, \quad u \in \widetilde{H}^{1}(\Omega), k \in[-\pi, \pi), \varepsilon>0 .
$$

Relations (6.7) and (2.6) imply that

$$
a_{1}(k)[u, u]+\varepsilon^{2} \int_{\Omega} Q(\mathbf{x})|u|^{2} d \mathbf{x} \geq\left(c_{0} k^{2}+c_{3} \varepsilon^{2}\right)\|u\|_{L_{2}(\Omega)}^{2}, \quad u \in \widetilde{H}^{1}(\Omega), k \in[-\pi, \pi), \varepsilon>0 .
$$

This yields (6.2).

Finally, by (5.1) and (2.12),

$$
\begin{aligned}
s(k, \varepsilon)[\omega, \omega] & \geq c_{0} \int_{\mathbb{R}}\left(k^{2}\left|\omega\left(x_{2}\right)\right|^{2}+\varepsilon^{2}\left|D_{2} \omega\left(x_{2}\right)\right|^{2}\right) d x_{2} \geq \\
& \geq c_{0} k^{2}\|\omega\|_{L_{2}(\mathbb{R})}^{2}, \quad \omega \in H^{1}(\mathbb{R}), k \in[-\pi, \pi), \quad \varepsilon>0 .
\end{aligned}
$$

From (6.9) and (2.16) it follows that

$$
s(k, \varepsilon)[\omega, \omega]+\varepsilon^{2} \int_{\mathbb{R}} Q^{0}\left(x_{2}\right)|\omega|^{2} d x_{2} \geq\left(c_{0} k^{2}+c_{3} \varepsilon^{2}\right)\|\omega\|_{L_{2}(\mathbb{R})}^{2}, \quad \omega \in H^{1}(\mathbb{R}), k \in[-\pi, \pi), \quad \varepsilon>0,
$$

which implies (6.3).

Lemma 6 We have

$$
\begin{gathered}
\left\|\left(D_{1}+k\right)\left(A(k, \varepsilon)+\varepsilon^{2} Q\right)^{-\frac{1}{2}}\right\|_{L_{2}(\Omega) \rightarrow L_{2}(\Omega)} \leq c_{0}^{-\frac{1}{2}}, \quad k \in[-\pi, \pi), \quad \varepsilon>0, \\
\left\|\varepsilon D_{2}\left(A(k, \varepsilon)+\varepsilon^{2} Q\right)^{-\frac{1}{2}}\right\|_{L_{2}(\Omega) \rightarrow L_{2}(\Omega)} \leq c_{0}^{-\frac{1}{2}}, \quad k \in[-\pi, \pi), \quad \varepsilon>0 \\
\left\|\left(D_{1}+k\right) A_{1}(k)^{-\frac{1}{2}}\right\|_{L_{2}(\Omega) \rightarrow L_{2}(\Omega)} \leq c_{0}^{-\frac{1}{2}}, \quad k \in[-\pi, \pi), k \neq 0 \\
\left\|\varepsilon D_{2}\left(S(k, \varepsilon)+\varepsilon^{2} Q^{0}\right)^{-\frac{1}{2}} P\right\|_{L_{2}(\Omega) \rightarrow L_{2}(\Omega)} \leq c_{0}^{-\frac{1}{2}}, \quad k \in[-\pi, \pi) .
\end{gathered}
$$


Proof. By (2.1) and (3.11), we obtain

$$
\begin{aligned}
c_{0} \int_{\Omega}\left(\left|\left(D_{1}+k\right) u\right|^{2}+\varepsilon^{2}\left|D_{2} u\right|^{2}\right) d \mathbf{x} & \leq a(k, \varepsilon)[u, u]+\varepsilon^{2} \int_{\Omega} Q(\mathbf{x})|u|^{2} d \mathbf{x}= \\
& =\left\|\left(A(k, \varepsilon)+\varepsilon^{2} Q\right)^{\frac{1}{2}} u\right\|_{L_{2}(\Omega)}^{2}, \quad u \in \widetilde{H}^{1}(\Omega), k \in[-\pi, \pi), \varepsilon>0 .
\end{aligned}
$$

Substituting $u=\left(A(k, \varepsilon)+\varepsilon^{2} Q\right)^{-\frac{1}{2}} v, v \in L_{2}(\Omega)$, in (6.15), we derive (6.11) and (6.12).

Similarly, (6.6) implies (6.13). Finally, (6.14) follows from the estimate

$$
\begin{aligned}
c_{0} \int_{\mathbb{R}}\left(k^{2}|\omega|^{2}+\varepsilon^{2}\left|D_{2} \omega\right|^{2}\right) d x_{2} & \leq s(k, \varepsilon)[\omega, \omega]+\varepsilon^{2} \int_{\mathbb{R}} Q^{0}\left(x_{2}\right)|\omega|^{2} d x_{2}= \\
& =\left\|\left(S(k, \varepsilon)+\varepsilon^{2} Q^{0}\right)^{\frac{1}{2}} \omega\right\|_{L_{2}(\mathbb{R})}^{2}, \quad \omega \in H^{1}(\mathbb{R}), \quad k \in[-\pi, \pi), \quad \varepsilon>0,
\end{aligned}
$$

see (6.9).

\subsection{Estimates for generalized resolvents multiplied by projections}

Recall that $F(k)$ denotes the spectral projection of the operator $A_{1}(k)$ for the interval $[0, \delta]$, and $P$ denotes the orthogonal projection of $L_{2}(\Omega)$ onto the subspace $\mathfrak{N}$ of functions depending only on $x_{2}$.

Lemma 7 Let $t=|k| \leq t_{0}, \varepsilon>0$. Then

$$
\begin{gathered}
\left\|F(k)^{\perp}\left(A(k, \varepsilon)+\varepsilon^{2} Q\right)^{-\frac{1}{2}}\right\|_{L_{2}(\Omega) \rightarrow L_{2}(\Omega)}=\left\|\left(A(k, \varepsilon)+\varepsilon^{2} Q\right)^{-\frac{1}{2}} F(k)^{\perp}\right\|_{L_{2}(\Omega) \rightarrow L_{2}(\Omega)} \leq \delta^{-\frac{1}{2}}, \\
\left\|\left(A(k, \varepsilon)+\varepsilon^{2} Q\right)^{-\frac{1}{2}} P^{\perp}\right\|_{L_{2}(\Omega) \rightarrow L_{2}(\Omega)} \leq C_{3}:=\delta^{-\frac{1}{2}}+C_{1} c_{0}^{-\frac{1}{2}}, \\
\left\|F(k)^{\perp}\left(S(k, \varepsilon)+\varepsilon^{2} Q^{0}\right)^{-\frac{1}{2}} P\right\|_{L_{2}(\Omega) \rightarrow L_{2}(\Omega)} \leq C_{4}:=C_{1} c_{0}^{-\frac{1}{2}} .
\end{gathered}
$$

Proof. By (3.11), (4.1) and (2.6), we have

$$
a_{1}(k)[u, u]+\varepsilon^{2} c_{3}\|u\|_{L_{2}(\Omega)}^{2} \leq a(k, \varepsilon)[u, u]+\varepsilon^{2}(Q u, u)_{L_{2}(\Omega)}, \quad u \in \widetilde{H}^{1}(\Omega), k \in[-\pi, \pi), \varepsilon>0 .
$$

Hence, we get:

$$
\left\|\left(A_{1}(k)+\varepsilon^{2} c_{3} I\right)^{\frac{1}{2}}\left(A(k, \varepsilon)+\varepsilon^{2} Q\right)^{-\frac{1}{2}}\right\|_{L_{2}(\Omega) \rightarrow L_{2}(\Omega)} \leq 1, \quad k \in[-\pi, \pi), \varepsilon>0 .
$$

Therefore,

$$
\begin{aligned}
&\left\|F(k)^{\perp}\left(A(k, \varepsilon)+\varepsilon^{2} Q\right)^{-\frac{1}{2}}\right\|_{L_{2}(\Omega) \rightarrow L_{2}(\Omega)} \leq \\
& \leq\left\|F(k)^{\perp}\left(A_{1}(k)+\varepsilon^{2} c_{3} I\right)^{-\frac{1}{2}}\right\|_{L_{2}(\Omega) \rightarrow L_{2}(\Omega)}\left\|\left(A_{1}(k)+\varepsilon^{2} c_{3} I\right)^{\frac{1}{2}}\left(A(k, \varepsilon)+\varepsilon^{2} Q\right)^{-\frac{1}{2}}\right\| \leq \\
& \leq\left\|F(k)^{\perp}\left(A_{1}(k)+\varepsilon^{2} c_{3} I\right)^{-\frac{1}{2}}\right\|_{L_{2}(\Omega) \rightarrow L_{2}(\Omega)} \leq \delta^{-\frac{1}{2}},
\end{aligned}
$$

which implies (6.16).

To prove (6.17), we use (4.29), which gives $P^{\perp}=F(k)^{\perp}+t \Phi(k)$, and (4.33). Then, by (6.16) and (6.1), we get

$$
\begin{aligned}
& \left\|\left(A(k, \varepsilon)+\varepsilon^{2} Q\right)^{-\frac{1}{2}} P^{\perp}\right\|_{L_{2}(\Omega) \rightarrow L_{2}(\Omega)}=\left\|\left(A(k, \varepsilon)+\varepsilon^{2} Q\right)^{-\frac{1}{2}}\left(F(k)^{\perp}+t \Phi(k)\right)\right\| \leq \\
& \leq \delta^{-\frac{1}{2}}+C_{1} t\left(c_{0} t^{2}+c_{3} \varepsilon^{2}\right)^{-\frac{1}{2}} \leq \delta^{-\frac{1}{2}}+C_{1} c_{0}^{-\frac{1}{2}}=C_{3}, \quad t=|k| \leq t_{0}, \varepsilon>0 .
\end{aligned}
$$

Finally, note that $P^{\perp}\left(S(k, \varepsilon)+\varepsilon^{2} Q^{0}\right)^{-\frac{1}{2}} P=0$. Then, by (4.29), $F(k)^{\perp}=P^{\perp}-t \Phi(k)$, and from (4.33) and (6.3) we obtain

$$
\begin{aligned}
& \left\|F(k)^{\perp}\left(S(k, \varepsilon)+\varepsilon^{2} Q^{0}\right)^{-\frac{1}{2}} P\right\|_{L_{2}(\Omega) \rightarrow L_{2}(\Omega)}=\left\|t \Phi(k)\left(S(k, \varepsilon)+\varepsilon^{2} Q^{0}\right)^{-\frac{1}{2}} P\right\| \leq \\
& \leq C_{1} t\left(c_{0} t^{2}+c_{3} \varepsilon^{2}\right)^{-\frac{1}{2}} \leq C_{1} c_{0}^{-\frac{1}{2}}=C_{4}, \quad t=|k| \leq t_{0}, \quad \varepsilon>0 .
\end{aligned}
$$

Lemma 7 together with Lemma 5 imply the following corollary. 
Corollary 8 Let $t=|k| \leq t_{0}, \varepsilon>0$. Then

$$
\begin{gathered}
\left\|\left(A(k, \varepsilon)+\varepsilon^{2} Q\right)^{-1} P^{\perp}\right\|_{L_{2}(\Omega) \rightarrow L_{2}(\Omega)} \leq C_{3} c_{3}^{-\frac{1}{2}} \varepsilon^{-1}, \\
\left\|F(k)^{\perp}\left(S(k, \varepsilon)+\varepsilon^{2} Q^{0}\right)^{-1} P\right\|_{L_{2}(\Omega) \rightarrow L_{2}(\Omega)} \leq C_{4} c_{3}^{-\frac{1}{2}} \varepsilon^{-1} .
\end{gathered}
$$

Indeed, (6.20) follows from (6.1) and (6.17), while (6.21) is a consequence of (6.3) and (6.18).

\subsection{One more reduction}

Now we represent the operator under the norm sign in (5.5) as

$$
\begin{aligned}
\left(A(k, \varepsilon)+\varepsilon^{2} Q\right)^{-1} & -\left(S(k, \varepsilon)+\varepsilon^{2} Q^{0}\right)^{-1} P= \\
& =\left(A(k, \varepsilon)+\varepsilon^{2} Q\right)^{-1} P^{\perp}-F(k)^{\perp}\left(S(k, \varepsilon)+\varepsilon^{2} Q^{0}\right)^{-1} P+\mathcal{J}(k, \varepsilon),
\end{aligned}
$$

where

$$
\mathcal{J}(k, \varepsilon):=\left(A(k, \varepsilon)+\varepsilon^{2} Q\right)^{-1} P-F(k)\left(S(k, \varepsilon)+\varepsilon^{2} Q^{0}\right)^{-1} P .
$$

Estimates (6.20), (6.21) and identity (6.22) show that Theorem 4 is a direct consequence of the following proposition.

Proposition 9 Suppose that conditions of Theorem 4 are satisfied. Let $\mathcal{J}(k, \varepsilon)$ be the operator defined by (6.23). Then

$$
\|\mathcal{J}(k, \varepsilon)\|_{L_{2}(\Omega) \rightarrow L_{2}(\Omega)} \leq \widehat{C} \varepsilon^{-1}, \quad|k| \leq t_{0}, \quad 0<\varepsilon \leq 1 .
$$

The constant $\widehat{C}$ depends only on $c_{j}, j=0, \ldots, 5$.

Clearly, the constant $\widetilde{C}$ in (5.5) is given by $\widetilde{C}=\left(C_{3}+C_{4}\right) c_{3}^{-1 / 2}+\widehat{C}$. Proposition 9 (and Theorem 4 with it) will be proved in Section 8 ,

\section{Estimates for commutators}

For the proof of Proposition 9, we need estimates for the commutators of the operator $D_{2}$ with the generalized resolvent of $A(k, \varepsilon)$ and with the projection $F(k)$.

\subsection{The commutator of $D_{2}$ and $\left(A(k, \varepsilon)+\varepsilon^{2} Q\right)^{-1}$}

The following fact can be proved by analogy with the proof of Theorem 8.8 in [GT].

Lemma 10 Suppose that $g_{j}(\mathbf{x}), j=1,2$, satisfy conditions of the form (2.1) and (2.3) in $\Omega=(0,1) \times \mathbb{R}$. Suppose that $Q(\mathbf{x})$ satisfies conditions of the form (2.6) and (2.7) in $\Omega$. Let $u \in \widetilde{H}^{1}(\Omega)$ be a (weak) solution of the equation

$$
\left(D_{1}+k\right) g_{1}(\mathbf{x})\left(D_{1}+k\right) u+\varepsilon^{2} D_{2} g_{2}(\mathbf{x}) D_{2} u+\varepsilon^{2} Q(\mathbf{x}) u=f(\mathbf{x}),
$$

where $f \in L_{2}(\Omega)$, which means that $u$ satisfies the identity

$$
\int_{\Omega}\left(g_{1}(\mathbf{x})\left(D_{1}+k\right) u \overline{\left(D_{1}+k\right) w}+\varepsilon^{2} g_{2}(\mathbf{x}) D_{2} u \overline{D_{2} w}+\varepsilon^{2} Q(\mathbf{x}) u \bar{w}\right) d \mathbf{x}=\int_{\Omega} f \bar{w} d \mathbf{x}, \forall w \in \widetilde{H}^{1}(\Omega) .
$$

Then

$$
D_{2} u \in \widetilde{H}^{1}(\Omega) .
$$

Let us prove the following lemma. 
Lemma 11 Let $|k| \leq t_{0}, \varepsilon>0$. The operator

$$
K_{1}(k, \varepsilon):=\operatorname{clos}\left[\left(A(k, \varepsilon)+\varepsilon^{2} Q\right)^{-1}, D_{2}\right]
$$

is bounded in $L_{2}(\Omega)$, and satisfies the estimate

$$
\left\|K_{1}(k, \varepsilon) P^{\perp}\right\|_{L_{2}(\Omega) \rightarrow L_{2}(\Omega)}=\left\|P^{\perp} K_{1}(k, \varepsilon)\right\|_{L_{2}(\Omega) \rightarrow L_{2}(\Omega)} \leq C_{5}\left(c_{0} k^{2}+c_{3} \varepsilon^{2}\right)^{-\frac{1}{2}},
$$

where the constant $C_{5}$ is defined below in (7.9) and depends only on $c_{0}, c_{1}, c_{2}, c_{3}, c_{5}$.

Proof. Let $u, v \in \widetilde{H}^{1}(\Omega)$ be such that $D_{2} u, D_{2} v \in \widetilde{H}^{1}(\Omega)$. Then we have

$$
\begin{aligned}
a(k, \varepsilon)[ & \left.D_{2} u, v\right]+\varepsilon^{2}\left(Q D_{2} u, v\right)_{L_{2}(\Omega)}-a(k, \varepsilon)\left[u, D_{2} v\right]-\varepsilon^{2}\left(Q u, D_{2} v\right)_{L_{2}(\Omega)}= \\
= & \int_{\Omega}\left(g_{1}(\mathbf{x})\left(\left(D_{1}+k\right) D_{2} u\right) \overline{\left(D_{1}+k\right) v}+\varepsilon^{2} g_{2}(\mathbf{x}) D_{2}^{2} u \overline{D_{2} v}\right) d \mathbf{x}- \\
& -\int_{\Omega}\left(g_{1}(\mathbf{x})\left(D_{1}+k\right) u\left(\overline{\left(D_{1}+k\right) D_{2} v}\right)+\varepsilon^{2} g_{2}(\mathbf{x}) D_{2} u \overline{D_{2}^{2} v}\right) d \mathbf{x}+\varepsilon^{2} \int_{\Omega} Q(\mathbf{x})\left(\left(D_{2} u\right) \bar{v}-u \overline{D_{2} v}\right) d \mathbf{x}= \\
= & -\int_{\Omega}\left(\left(D_{2} g_{1}\right)(\mathbf{x})\left(D_{1}+k\right) u \overline{\left(D_{1}+k\right) v}+\varepsilon^{2}\left(D_{2} g_{2}\right)(\mathbf{x}) D_{2} u \overline{D_{2} v}\right) d \mathbf{x}-\varepsilon^{2} \int_{\Omega}\left(D_{2} Q\right)(\mathbf{x}) u \bar{v} d \mathbf{x} .
\end{aligned}
$$

Here we have integrated by parts in direction $x_{2}$. Now we put

$$
u=\left(A(k, \varepsilon)+\varepsilon^{2} Q\right)^{-1} \varphi, \quad v=\left(A(k, \varepsilon)+\varepsilon^{2} Q\right)^{-1} \psi, \quad \varphi, \psi \in L_{2}(\Omega) .
$$

By Lemma 10, we have $u, v \in \widetilde{H}^{1}(\Omega), D_{2} u, D_{2} v \in \widetilde{H}^{1}(\Omega)$.

Then from (7.6) we obtain

$$
\begin{aligned}
\left(D_{2}(A(k, \varepsilon)\right. & \left.\left.+\varepsilon^{2} Q\right)^{-1} \varphi, \psi\right)_{L_{2}(\Omega)}-\left(\varphi, D_{2}\left(A(k, \varepsilon)+\varepsilon^{2} Q\right)^{-1} \psi\right)_{L_{2}(\Omega)}= \\
= & -\int_{\Omega}\left(D_{2} g_{1}\right)(\mathbf{x})\left(\left(D_{1}+k\right)\left(A(k, \varepsilon)+\varepsilon^{2} Q\right)^{-1} \varphi\right)\left(\overline{\left(D_{1}+k\right)\left(A(k, \varepsilon)+\varepsilon^{2} Q\right)^{-1} \psi}\right) d \mathbf{x}- \\
& -\int_{\Omega} \varepsilon^{2}\left(D_{2} g_{2}\right)(\mathbf{x})\left(D_{2}\left(A(k, \varepsilon)+\varepsilon^{2} Q\right)^{-1} \varphi\right)\left(\overline{D_{2}\left(A(k, \varepsilon)+\varepsilon^{2} Q\right)^{-1} \psi}\right) d \mathbf{x}- \\
& -\int_{\Omega} \varepsilon^{2}\left(D_{2} Q\right)(\mathbf{x})\left(\left(A(k, \varepsilon)+\varepsilon^{2} Q\right)^{-1} \varphi\right)\left(\overline{\left(A(k, \varepsilon)+\varepsilon^{2} Q\right)^{-1} \psi}\right) d \mathbf{x} .
\end{aligned}
$$

It follows that

$$
\begin{aligned}
K_{1}(k, \varepsilon)= & -\left(\left(D_{1}+k\right)\left(A(k, \varepsilon)+\varepsilon^{2} Q\right)^{-1}\right)^{*}\left[D_{2} g_{1}\right]\left(\left(D_{1}+k\right)\left(A(k, \varepsilon)+\varepsilon^{2} Q\right)^{-1}\right)- \\
& -\left(\varepsilon D_{2}\left(A(k, \varepsilon)+\varepsilon^{2} Q\right)^{-1}\right)^{*}\left[D_{2} g_{2}\right]\left(\varepsilon D_{2}\left(A(k, \varepsilon)+\varepsilon^{2} Q\right)^{-1}\right)- \\
& -\varepsilon^{2}\left(A(k, \varepsilon)+\varepsilon^{2} Q\right)^{-1}\left[D_{2} Q\right]\left(A(k, \varepsilon)+\varepsilon^{2} Q\right)^{-1} .
\end{aligned}
$$

By (2.3), (2.7), (6.1), (6.11) and (6.12), the operator on the right is bounded in $L_{2}(\Omega)$.

Next, from (2.3), (2.7), (6.1), (6.11), (6.12), (6.17) and (7.8) it follows that

$$
\begin{aligned}
\| K_{1}(k, \varepsilon) P^{\perp} & \|_{L_{2}(\Omega) \rightarrow L_{2}(\Omega)} \leq \\
\leq & \left\|\left(D_{1}+k\right)\left(A(k, \varepsilon)+\varepsilon^{2} Q\right)^{-\frac{1}{2}}\right\|_{L_{2}(\Omega) \rightarrow L_{2}(\Omega)}^{2}\left\|\left(A(k, \varepsilon)+\varepsilon^{2} Q\right)^{-\frac{1}{2}}\right\|_{L_{2}(\Omega) \rightarrow L_{2}(\Omega)} \times \\
& \times\left\|D_{2} g_{1}\right\|_{L_{\infty}}\left\|\left(A(k, \varepsilon)+\varepsilon^{2} Q\right)^{-\frac{1}{2}} P^{\perp}\right\|_{L_{2}(\Omega) \rightarrow L_{2}(\Omega)}+ \\
+ & \left\|\varepsilon D_{2}\left(A(k, \varepsilon)+\varepsilon^{2} Q\right)^{-\frac{1}{2}}\right\|_{L_{2}(\Omega) \rightarrow L_{2}(\Omega)}^{2}\left\|\left(A(k, \varepsilon)+\varepsilon^{2} Q\right)^{-\frac{1}{2}}\right\|_{L_{2}(\Omega) \rightarrow L_{2}(\Omega)} \times \\
& \times\left\|D_{2} g_{2}\right\|_{L_{\infty}}\left\|\left(A(k, \varepsilon)+\varepsilon^{2} Q\right)^{-\frac{1}{2}} P^{\perp}\right\|_{L_{2}(\Omega) \rightarrow L_{2}(\Omega)}+ \\
& +\varepsilon^{2}\left\|\left(A(k, \varepsilon)+\varepsilon^{2} Q\right)^{-1}\right\|_{L_{2}(\Omega) \rightarrow L_{2}(\Omega)}^{\frac{3}{2}}\left\|D_{2} Q\right\|_{L_{\infty}}\left\|\left(A(k, \varepsilon)+\varepsilon^{2} Q\right)^{-\frac{1}{2}} P^{\perp}\right\|_{L_{2}(\Omega) \rightarrow L_{2}(\Omega)} \leq \\
\leq & 2 c_{0}^{-1}\left(c_{0} k^{2}+c_{3} \varepsilon^{2}\right)^{-\frac{1}{2}} c_{2} C_{3}+\varepsilon^{2}\left(c_{0} k^{2}+c_{3} \varepsilon^{2}\right)^{-\frac{3}{2}} c_{5} C_{3},
\end{aligned}
$$

which implies (7.5) with

$$
C_{5}=2 c_{0}^{-1} c_{2} C_{3}+c_{3}^{-1} c_{5} C_{3}
$$




\subsection{The commutator of $D_{2}$ and $F(k)$}

In order to calculate the commutator $\left[D_{2}, F(k)\right]$, we represent the spectral projection $F(k)$ as the integral of the resolvent $\left(A_{1}(k)-z I\right)^{-1}$ over the contour $\Gamma_{\delta}$ that envelopes the real interval $[0, \delta]$ equidistantly with distance $\delta$. Recall that, for $|k| \leq t_{0}$, the interval $(\delta, 3 \delta)$ is free of the spectrum of $A_{1}(k)$; therefore,

$$
\left\|\left(A_{1}(k)-z I\right)^{-1}\right\|_{L_{2}(\Omega) \rightarrow L_{2}(\Omega)} \leq \delta^{-1}, \quad|k| \leq t_{0}, \quad z \in \Gamma_{\delta} .
$$

We have the following representation

$$
F(k)=-\frac{1}{2 \pi i} \int_{\Gamma_{\delta}}\left(A_{1}(k)-z I\right)^{-1} d z .
$$

Lemma 12 Let $t=|k| \leq t_{0}$ and $\varepsilon>0$. Then the operator

$$
K_{2}(k):=\cos \left[D_{2}, F(k)\right]
$$

is bounded in $L_{2}(\Omega)$, and

$$
\begin{gathered}
\left\|K_{2}(k)\right\|_{L_{2}(\Omega) \rightarrow L_{2}(\Omega)} \leq C_{6}, \\
\left\|K_{2}(k) F(k)\right\|_{L_{2}(\Omega) \rightarrow L_{2}(\Omega)} \leq C_{7} t,
\end{gathered}
$$

where the constants $C_{6}$ and $C_{7}$ are defined below in 7.21) and (7.27) respectively. These constants depend only on $c_{0}, c_{1}, c_{2}$.

Proof. We start with calculating the operator

$$
K_{3}(k, z):=\operatorname{clos}\left[D_{2},\left(A_{1}(k)-z I\right)^{-1}\right] .
$$

Suppose that functions $u, v \in \operatorname{Dom} a_{1}(k)$ are such that $D_{2} u, D_{2} v \in \operatorname{Dom} a_{1}(k)$. Then

$$
\begin{aligned}
& a_{1}(k)\left[D_{2} u, v\right]-z\left(D_{2} u, v\right)_{L_{2}(\Omega)}-a_{1}(k)\left[u, D_{2} v\right]+z\left(u, D_{2} v\right)_{L_{2}(\Omega)}= \\
& =\int_{\Omega}\left(g_{1}(\mathbf{x})\left(\left(D_{1}+k\right) D_{2} u\right) \overline{\left(D_{1}+k\right) v}-g_{1}(\mathbf{x})\left(\left(D_{1}+k\right) u\right) \overline{\left(D_{1}+k\right) D_{2} v}\right) d \mathbf{x}+ \\
& \quad+z \int_{\Omega}\left(u \overline{D_{2} v}-\left(D_{2} u\right) \bar{v}\right) d \mathbf{x}= \\
& =-\int_{\Omega}\left(D_{2} g_{1}\right)(\mathbf{x})\left(\left(D_{1}+k\right) u\right) \overline{\left(D_{1}+k\right) v} d \mathbf{x} .
\end{aligned}
$$

We have integrated by parts in direction $x_{2}$. We put $u=\left(A_{1}(k)-z I\right)^{-1} \varphi, v=\left(A_{1}(k)-z I\right)^{-1} \psi$, where $\varphi, \psi \in \widetilde{C}_{0}^{\infty}(\Omega)$. Here $\widetilde{C}_{0}^{\infty}(\Omega)$ is a class of infinitely smooth functions in $\Omega$ periodic in $x_{1}$ (which means that 1-periodic extension of them to $\mathbb{R}^{2}$ is $C^{\infty}$ in $\mathbb{R}^{2}$ ) and equal to zero for large $\left|x_{2}\right|$. By using (2.3), it is easily seen that $u$ and $v$ satisfy the required conditions. Then from (7.16) we obtain for all $\varphi, \psi \in \widetilde{C}_{0}^{\infty}(\Omega)$ :

$$
\begin{aligned}
\left(D _ { 2 } \left(A_{1}(k)\right.\right. & \left.-z I)^{-1} \varphi, \psi\right)_{L_{2}(\Omega)}-\left(\varphi, D_{2}\left(A_{1}(k)-z I\right)^{-1} \psi\right)_{L_{2}(\Omega)}= \\
& =-\int_{\Omega}\left(D_{2} g_{1}\right)(\mathbf{x})\left(\left(D_{1}+k\right)\left(A_{1}(k)-z I\right)^{-1} \varphi\right)\left(\overline{\left(D_{1}+k\right)\left(A_{1}(k)-z I\right)^{-1} \psi}\right) d \mathbf{x} .
\end{aligned}
$$

Since $\widetilde{C}_{0}^{\infty}(\Omega)$ is dense in $L_{2}(\Omega)$, it follows that

$$
K_{3}(k, z)=-\left(\left(D_{1}+k\right)\left(A_{1}(k)-z I\right)^{-1}\right)^{*}\left[D_{2} g_{1}\right]\left(\left(D_{1}+k\right)\left(A_{1}(k)-z I\right)^{-1}\right) .
$$

Then, by (2.3) and (6.13), we have

$$
\begin{aligned}
\left\|K_{3}(k, z)\right\|_{L_{2}(\Omega) \rightarrow L_{2}(\Omega)} & \leq\left\|D_{2} g_{1}\right\|_{L_{\infty}}\left\|\left(D_{1}+k\right)\left(A_{1}(k)-z I\right)^{-1}\right\|_{L_{2}(\Omega) \rightarrow L_{2}(\Omega)}^{2} \leq \\
& \leq c_{2}\left\|\left(D_{1}+k\right) A_{1}(k)^{-\frac{1}{2}}\right\|_{L_{2}(\Omega) \rightarrow L_{2}(\Omega)}^{2}\left\|A_{1}(k)^{\frac{1}{2}}\left(A_{1}(k)-z I\right)^{-1}\right\|_{L_{2}(\Omega) \rightarrow L_{2}(\Omega)}^{2} \leq \\
& \leq c_{2} c_{0}^{-1}\left\|A_{1}(k)^{\frac{1}{2}}\left(A_{1}(k)-z I\right)^{-1}\right\|_{L_{2}(\Omega) \rightarrow L_{2}(\Omega)}^{2} .
\end{aligned}
$$


Next, for $|k| \leq t_{0}$ we have:

$$
\left\|A_{1}(k)^{\frac{1}{2}}\left(A_{1}(k)-z I\right)^{-1}\right\|_{L_{2}(\Omega) \rightarrow L_{2}(\Omega)} \leq \max \left\{\lambda^{\frac{1}{2}}|\lambda-z|^{-1}: \lambda \in[0, \delta] \cup[3 \delta, \infty), z \in \Gamma_{\delta}\right\}=: c(\delta) .
$$

Hence,

$$
\left\|K_{3}(k, z)\right\|_{L_{2}(\Omega) \rightarrow L_{2}(\Omega)} \leq c_{2} c_{0}^{-1} c(\delta)^{2}, \quad z \in \Gamma_{\delta},|k| \leq t_{0} .
$$

Now, from (7.11), (7.12) and (7.15) it follows that

$$
K_{2}(k)=-\frac{1}{2 \pi i} \int_{\Gamma_{\delta}} K_{3}(k, z) d z .
$$

Combining (7.19) with (7.20), we obtain

$$
\left\|K_{2}(k)\right\|_{L_{2}(\Omega) \rightarrow L_{2}(\Omega)} \leq(2 \delta+2 \pi \delta)(2 \pi)^{-1} c_{2} c_{0}^{-1} c(\delta)^{2}=: C_{6}, \quad|k| \leq t_{0},
$$

which proves (7.13).

Next, by (7.17), (2.3), (6.13), (7.18), (7.10), we have

$$
\begin{aligned}
& \left\|K_{3}(k, z) F(k)\right\|_{L_{2}(\Omega) \rightarrow L_{2}(\Omega)} \leq \\
& \leq\left\|D_{2} g_{1}\right\|_{L_{\infty}}\left\|\left(D_{1}+k\right)\left(A_{1}(k)-z I\right)^{-1}\right\|_{L_{2}(\Omega) \rightarrow L_{2}(\Omega)} \times \\
& \quad \times\left\|\left(D_{1}+k\right)\left(A_{1}(k)-z I\right)^{-1} F(k)\right\|_{L_{2}(\Omega) \rightarrow L_{2}(\Omega)} \leq \\
& \leq c_{2}\left\|\left(D_{1}+k\right) A_{1}(k)^{-\frac{1}{2}}\right\|_{L_{2}(\Omega) \rightarrow L_{2}(\Omega)}^{2}\left\|A_{1}(k)^{\frac{1}{2}}\left(A_{1}(k)-z I\right)^{-1}\right\|_{L_{2}(\Omega) \rightarrow L_{2}(\Omega)} \times \\
& \quad \times\left\|\left(A_{1}(k)-z I\right)^{-1}\right\|_{L_{2}(\Omega) \rightarrow L_{2}(\Omega)}\left\|A_{1}(k)^{\frac{1}{2}} F(k)\right\|_{L_{2}(\Omega) \rightarrow L_{2}(\Omega)} \\
& \leq c_{2} c_{0}^{-1} c(\delta) \delta^{-1}\left\|A_{1}(k)^{\frac{1}{2}} F(k)\right\|_{L_{2}(\Omega) \rightarrow L_{2}(\Omega)}, \quad|k| \leq t_{0}, z \in \Gamma_{\delta} .
\end{aligned}
$$

Recalling (4.31) and (4.34), we obtain

$$
\begin{aligned}
\left\|A_{1}(k)^{\frac{1}{2}} F(k) u\right\|_{L_{2}(\Omega)}^{2} & =\left(A_{1}(k) F(k) u, u\right)_{L_{2}(\Omega)}=\left(\left(t^{2} S P+t^{3} \Psi(k)\right) u, u\right)_{L_{2}(\Omega)} \leq \\
& \leq\left(t^{2}\|S\|_{\mathfrak{N} \rightarrow \mathfrak{N}}+C_{2} t^{3}\right)\|u\|_{L_{2}(\Omega)}^{2}, \quad t=|k| \leq t_{0} .
\end{aligned}
$$

Since $S$ is the operator of multiplication by the function $g_{1}^{0}\left(x_{2}\right)$, by (2.12), we have

$$
\|S\|_{\mathfrak{N} \rightarrow \mathfrak{N}} \leq c_{1} .
$$

From (7.23) and (7.24) it follows that

$$
\left\|A_{1}(k)^{\frac{1}{2}} F(k)\right\|_{L_{2}(\Omega) \rightarrow L_{2}(\Omega)} \leq t\left(c_{1}+C_{2} t_{0}\right)^{\frac{1}{2}}, \quad t=|k| \leq t_{0} .
$$

Combining (7.22) and (7.25), we arrive at the estimate

$$
\left\|K_{3}(k, z) F(k)\right\|_{L_{2}(\Omega) \rightarrow L_{2}(\Omega)} \leq c_{2} c_{0}^{-1} c(\delta) \delta^{-1}\left(c_{1}+C_{2} t_{0}\right)^{\frac{1}{2}} t, \quad t=|k| \leq t_{0}, z \in \Gamma_{\delta} .
$$

Finally, from (7.26) and (7.20) we obtain (7.14) with the constant

$$
C_{7}=(2 \delta+2 \pi \delta)(2 \pi)^{-1} c_{2} c_{0}^{-1} c(\delta) \delta^{-1}\left(c_{1}+C_{2} t_{0}\right)^{\frac{1}{2}} .
$$

In the sequel, we shall need the following statement.

Lemma 13 We have

$$
F(k) \widetilde{H}^{1}(\Omega) \subset \widetilde{H}^{1}(\Omega) .
$$

Proof. Let $u \in \widetilde{H}^{1}(\Omega)$. Then, obviously, $u \in \operatorname{Dom} a_{1}(k)=\int_{\mathbb{R}} \oplus \widetilde{H}^{1}(0,1) d x_{2}$.

Since $F(k) \operatorname{Dom} a_{1}(k) \subset \operatorname{Dom} a_{1}(k)$, then $F(k) u \in \operatorname{Dom} a_{1}(k)$. This means that $F(k) u \in L_{2}(\Omega)$, $D_{1}(F(k) u) \in L_{2}(\Omega)$ and $F(k) u$ satisfies periodic boundary conditions on $\partial \Omega$. Next,

$$
D_{2}(F(k) u)=F(k) D_{2} u+\left[D_{2}, F(k)\right] u \in L_{2}(\Omega),
$$

because $D_{2} u \in L_{2}(\Omega)$ and the operators $F(k)$ and $\left[D_{2}, F(k)\right]$ are bounded in $L_{2}(\Omega)$ (see Lemma 12). It follows that $F(k) u \in \widetilde{H}^{1}(\Omega)$. 


\section{Proof of Proposition 9}

\subsection{Abstract lemma}

For the study of the operator (6.23), we wish to apply the identity

$$
\mathcal{J}(k, \varepsilon)=\left(A(k, \varepsilon)+\varepsilon^{2} Q\right)^{-1}\left(S(k, \varepsilon) P+\varepsilon^{2} Q^{0} P-A(k, \varepsilon) F(k)-\varepsilon^{2} Q F(k)\right)\left(S(k, \varepsilon)+\varepsilon^{2} Q^{0}\right)^{-1} P .
$$

However, the operator in the central brackets makes no sense. In order to obtain a precise version of (8.1), we need the following statement of abstract nature (see Lemma $7.1 \mathrm{in}[\mathrm{Su}]$ ).

Lemma 14 Let $\mathfrak{t}_{1}$ and $\mathfrak{t}_{2}$ be two closed and densely defined sesquilinear forms in a separable Hilbert space $\mathfrak{H}$. Suppose that both quadratic forms $\mathfrak{t}_{1}[u, u]$ and $\mathfrak{t}_{2}[u, u]$ are positive definite. Let $T_{1}$ and $T_{2}$ be the selfadjoint operators in $\mathfrak{H}$ corresponding to the forms $\mathfrak{t}_{1}$ and $\mathfrak{t}_{2}$ respectively. Let $\mathcal{P}$ and $\mathcal{Q}$ be some orthogonal projections in $\mathfrak{H}$. Let the following conditions be satisfied:

$1^{\circ} \cdot \mathfrak{d}:=\operatorname{Dom}_{1} \subset \operatorname{Dom}_{2} ;$

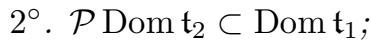

$3^{\circ} . \mathcal{Q} \mathfrak{d} \subset \mathfrak{d}$;

$4^{\circ} . \mathcal{P}$ commutes with $T_{2}$;

$5^{\circ}$. the following representation holds

$$
\mathfrak{t}_{2}[\mathcal{P} u, v]-\mathfrak{t}_{1}[\mathcal{Q} u, v]=\left(G_{0} u, G v\right)_{\mathfrak{G}}, \quad u, v \in \mathfrak{d},
$$

where $G_{0}, G: \mathfrak{H} \rightarrow \mathfrak{G}$ are densely defined linear operators acting from $\mathfrak{H}$ to some separable Hilbert space $\mathfrak{G}$. Moreover, $\mathfrak{d} \subset \operatorname{Dom} G_{0}, \mathfrak{d} \subset \operatorname{Dom} G$, and the operators

$$
G T_{1}^{-1}: \mathfrak{H} \rightarrow \mathfrak{G}, \quad G_{0} T_{2}^{-1} \mathcal{P}: \mathfrak{H} \rightarrow \mathfrak{G}
$$

are bounded. Then we have

$$
T_{1}^{-1} \mathcal{P}-\mathcal{Q} T_{2}^{-1} \mathcal{P}=\left(G T_{1}^{-1}\right)^{*}\left(G_{0} T_{2}^{-1} \mathcal{P}\right)
$$

Let $|k| \leq t_{0}, \varepsilon>0$. We shall apply Lemma 14 with

$$
\begin{aligned}
\mathfrak{H} & =L_{2}(\Omega), \quad \mathcal{P}=P, \quad \mathcal{Q}=F(k), \\
\mathfrak{t}_{1}[u, v] & =a(k, \varepsilon)[u, v]+\varepsilon^{2}(Q u, v)_{L_{2}(\Omega)}, \\
\mathfrak{t}_{2}[u, v] & =s(k, \varepsilon)[P u, P v]+\varepsilon^{2}\left(Q^{0} u, v\right)_{L_{2}(\Omega)}, \\
\mathfrak{d} & =\widetilde{H}^{1}(\Omega) \text { and } \quad \operatorname{Dom}_{2}=\left\{u \in L_{2}(\Omega): P u \in H^{1}(\mathbb{R})\right\} .
\end{aligned}
$$

Obviously, conditions $1^{\circ}, 2^{\circ}$ and $4^{\circ}$ are satisfied. Condition $3^{\circ}$ is also satisfied, by Lemma 13 .

Let us check condition $5^{\circ}$. The left-hand side of (8.2) takes the form

$$
\begin{aligned}
\mathcal{I}[u, v] & :=s(k, \varepsilon)[P u, P v]+\varepsilon^{2}\left(Q^{0} P u, v\right)_{L_{2}(\Omega)}-a(k, \varepsilon)[F(k) u, v]-\varepsilon^{2}(Q F(k) u, v)_{L_{2}(\Omega)}= \\
& =\mathcal{I}_{1}[u, v]+\mathcal{I}_{2}[u, v]+\mathcal{I}_{3}[u, v],
\end{aligned}
$$

where, in accordance with (3.11), (4.1) and (5.1),

$$
\begin{aligned}
& \mathcal{I}_{1}[u, v]=\varepsilon^{2}\left(Q^{0} P u-Q F(k) u, v\right)_{L_{2}(\Omega)}, \\
& \mathcal{I}_{2}[u, v]=t^{2}(S P u, P v)_{L_{2}(\Omega)}-a_{1}(k)[F(k) u, v] \\
& \mathcal{I}_{3}[u, v]=\left(g_{2}^{0} \varepsilon D_{2} P u, \varepsilon D_{2} P v\right)_{L_{2}(\Omega)}-\left(g_{2} \varepsilon D_{2} F(k) u, \varepsilon D_{2} v\right)_{L_{2}(\Omega)} .
\end{aligned}
$$




\subsection{The form $\mathcal{I}_{1}$}

We represent the form (8.5) as

$$
\mathcal{I}_{1}[u, v]=\mathcal{I}_{1}^{\prime}[u, v]+\mathcal{I}_{1}^{\prime \prime}[u, v]
$$

where

$$
\begin{aligned}
& \mathcal{I}_{1}^{\prime}[u, v]=\varepsilon^{2}\left(\left(Q^{0}-Q\right) P u, v\right)_{L_{2}(\Omega)}, \\
& \mathcal{I}_{1}^{\prime \prime}[u, v]=\varepsilon^{2}(Q(P-F(k)) u, v)_{L_{2}(\Omega)} .
\end{aligned}
$$

We have

$$
\begin{aligned}
\mathcal{I}_{1}^{\prime}[u, v] & =\varepsilon^{2}\left(Q^{0} P u, P v\right)_{L_{2}(\Omega)}-\varepsilon^{2}(Q P u, P v)_{L_{2}(\Omega)}-\varepsilon^{2}\left(Q P u, P^{\perp} v\right)_{L_{2}(\Omega)}= \\
& =-\varepsilon^{2}\left(Q P u, P^{\perp} v\right)_{L_{2}(\Omega)},
\end{aligned}
$$

since $P Q P=Q^{0} P$. Then

$$
\mathcal{I}_{1}^{\prime}[u, v]=\left(G_{01}^{\prime} u, G_{1}^{\prime} v\right)_{L_{2}(\Omega)},
$$

where $G_{01}^{\prime}=-\varepsilon^{\frac{3}{2}} Q P, G_{1}^{\prime}=\varepsilon^{\frac{1}{2}} P^{\perp}$. By (6.3) and (2.6), we obtain:

$$
\left\|G_{01}^{\prime}\left(S(k, \varepsilon) P+\varepsilon^{2} Q^{0}\right)^{-1} P\right\|_{L_{2}(\Omega) \rightarrow L_{2}(\Omega)} \leq \varepsilon^{\frac{3}{2}}\|Q\|_{L_{\infty}}\left(c_{0} k^{2}+c_{3} \varepsilon^{2}\right)^{-1} \leq c_{4} c_{3}^{-1} \varepsilon^{-\frac{1}{2}} .
$$

Using (6.20), we obtain that

$$
\begin{aligned}
\left\|G_{1}^{\prime}\left(A(k, \varepsilon)+\varepsilon^{2} Q\right)^{-1}\right\|_{L_{2}(\Omega) \rightarrow L_{2}(\Omega)} & \leq \varepsilon^{\frac{1}{2}}\left\|P^{\perp}\left(A(k, \varepsilon)+\varepsilon^{2} Q\right)^{-1}\right\|_{L_{2}(\Omega) \rightarrow L_{2}(\Omega)} \leq \\
& \leq C_{3} c_{3}^{-\frac{1}{2}} \varepsilon^{-\frac{1}{2}} .
\end{aligned}
$$

By (4.29), the form (8.10) can be written as

$$
\mathcal{I}_{1}^{\prime \prime}[u, v]=-\varepsilon^{2}(Q t \Phi(k) u, v)_{L_{2}(\Omega)}=\left(G_{01}^{\prime \prime} u, G_{1}^{\prime \prime} v\right)_{L_{2}(\Omega)},
$$

where $G_{01}^{\prime \prime}=-\varepsilon t^{\frac{1}{2}} Q \Phi(k), G_{1}^{\prime \prime}=\varepsilon t^{\frac{1}{2}} I$.

Using (4.33), (6.3) and (2.6), we have

$$
\begin{aligned}
\left\|G_{01}^{\prime \prime}\left(S(k, \varepsilon) P+\varepsilon^{2} Q^{0}\right)^{-1} P\right\|_{L_{2}(\Omega) \rightarrow L_{2}(\Omega)} & \leq \varepsilon t^{\frac{1}{2}}\|Q\|_{L_{\infty}} C_{1}\left(c_{0} k^{2}+c_{3} \varepsilon^{2}\right)^{-1} \leq \\
& \leq C_{1} c_{4} c_{0}^{-\frac{1}{4}} c_{3}^{-\frac{3}{4}} \varepsilon^{-\frac{1}{2}}
\end{aligned}
$$

Next, by (6.1),

$$
\left\|G_{1}^{\prime \prime}\left(A(k, \varepsilon)+\varepsilon^{2} Q\right)^{-1}\right\|_{L_{2}(\Omega) \rightarrow L_{2}(\Omega)} \leq \varepsilon t^{\frac{1}{2}}\left(c_{0} k^{2}+c_{3} \varepsilon^{2}\right)^{-1} \leq c_{0}^{-\frac{1}{4}} c_{3}^{-\frac{3}{4}} \varepsilon^{-\frac{1}{2}} .
$$

\subsection{The form $\mathcal{I}_{2}$}

As for the form (8.6), we apply (4.31):

$$
\begin{aligned}
\mathcal{I}_{2}[u, v] & =\left(\left(t^{2} S P-A_{1}(k) F(k)\right) u, v\right)_{L_{2}(\Omega)}=-t^{3}(\Psi(k) u, v)_{L_{2}(\Omega)}= \\
& =\left(G_{02} u, G_{2} v\right)_{L_{2}(\Omega)},
\end{aligned}
$$

where $G_{02}=-t^{\frac{3}{2}} \Psi(k), G_{2}=t^{\frac{3}{2}} I$. Then, by (4.34) and (6.3) we have

$$
\begin{aligned}
\left\|G_{02}\left(S(k, \varepsilon) P+\varepsilon^{2} Q^{0}\right)^{-1} P\right\|_{L_{2}(\Omega) \rightarrow L_{2}(\Omega)} & \leq t^{\frac{3}{2}} C_{2}\left(c_{0} k^{2}+c_{3} \varepsilon^{2}\right)^{-1} \leq \\
& \leq C_{2} c_{0}^{-\frac{3}{4}} c_{3}^{-\frac{1}{4}} \varepsilon^{-\frac{1}{2}}
\end{aligned}
$$

By (6.1),

$$
\left\|G_{2}\left(A(k, \varepsilon)+\varepsilon^{2} Q\right)^{-1}\right\|_{L_{2}(\Omega) \rightarrow L_{2}(\Omega)} \leq t^{\frac{3}{2}}\left(c_{0} k^{2}+c_{3} \varepsilon^{2}\right)^{-1} \leq c_{0}^{-\frac{3}{4}} c_{3}^{-\frac{1}{4}} \varepsilon^{-\frac{1}{2}} .
$$




\subsection{The form $\mathcal{I}_{3}$}

We rewrite the form (8.7) as

$$
\mathcal{I}_{3}[u, v]=\mathcal{I}_{3}^{\prime}[u, v]+\mathcal{I}_{4}[u, v]
$$

where

$$
\begin{aligned}
& \mathcal{I}_{3}^{\prime}[u, v]=-\left(g_{2} \varepsilon D_{2}(F(k)-P) u, \varepsilon D_{2} v\right)_{L_{2}(\Omega)}, \\
& \mathcal{I}_{4}[u, v]=\left(g_{2}^{0} \varepsilon D_{2} P u, \varepsilon D_{2} P v\right)_{L_{2}(\Omega)}-\left(g_{2} \varepsilon D_{2} P u, \varepsilon D_{2} v\right)_{L_{2}(\Omega)} .
\end{aligned}
$$

For the form (8.22) we have

$$
\mathcal{I}_{3}^{\prime}[u, v]=\left(G_{03} u, G_{3} v\right)_{L_{2}(\Omega)},
$$

where $G_{03}=-g_{2} \varepsilon^{\frac{1}{2}} D_{2}(F(k)-P), G_{3}=\varepsilon^{\frac{3}{2}} D_{2}$.

We represent $G_{03}$ as

$$
G_{03}=\widetilde{G}_{03}+\widehat{G}_{03}+\stackrel{\vee}{G_{03}}
$$

where

$$
\begin{aligned}
& \widetilde{G}_{03}=-g_{2} \varepsilon^{\frac{1}{2}}\left[D_{2}, F(k)\right] F(k), \\
& \widehat{G}_{03}=-g_{2} \varepsilon^{\frac{1}{2}}\left[D_{2}, F(k)\right] F(k)^{\perp}, \\
& \stackrel{\vee}{G}_{03}=-g_{2} \varepsilon^{\frac{1}{2}}(F(k)-P) D_{2}=-g_{2} \varepsilon^{\frac{1}{2}} t \Phi(k) D_{2} .
\end{aligned}
$$

Here we have taken (4.29) into account. From (2.1), (17.14) and (6.3) it follows that

$$
\begin{aligned}
& \left\|\widetilde{G}_{03}\left(S(k, \varepsilon) P+\varepsilon^{2} Q^{0}\right)^{-1} P\right\|_{L_{2}(\Omega) \rightarrow L_{2}(\Omega)} \leq \\
& \quad \leq\left\|g_{2}\right\|_{L_{\infty}} \varepsilon^{\frac{1}{2}}\left\|\left[D_{2}, F(k)\right] F(k)\right\|_{L_{2}(\Omega) \rightarrow L_{2}(\Omega)}\left\|\left(S(k, \varepsilon)+\varepsilon^{2} Q^{0}\right)^{-1}\right\|_{L_{2}(\Omega) \rightarrow L_{2}(\Omega)} \leq \\
& \quad \leq c_{1} \varepsilon^{\frac{1}{2}} C_{7} t\left(c_{0} k^{2}+c_{3} \varepsilon^{2}\right)^{-1} \leq c_{1} C_{7} c_{0}^{-\frac{1}{2}} c_{3}^{-\frac{1}{2}} \varepsilon^{-\frac{1}{2}} .
\end{aligned}
$$

Relations (2.1), (7.13) and (6.21) imply that

$$
\begin{aligned}
& \left\|\widehat{G}_{03}\left(S(k, \varepsilon) P+\varepsilon^{2} Q^{0}\right)^{-1} P\right\|_{L_{2}(\Omega) \rightarrow L_{2}(\Omega)} \leq \\
& \quad \leq\left\|g_{2}\right\|_{L_{\infty}} \varepsilon^{\frac{1}{2}}\left\|\left[D_{2}, F(k)\right]\right\|_{L_{2}(\Omega) \rightarrow L_{2}(\Omega)}\left\|F(k)^{\perp}\left(S(k, \varepsilon) P+\varepsilon^{2} Q^{0}\right)^{-1} P\right\| \leq \\
& \quad \leq c_{1} \varepsilon^{\frac{1}{2}} C_{6} C_{4} c_{3}^{-\frac{1}{2}} \varepsilon^{-1}=c_{1} C_{6} C_{4} c_{3}^{-\frac{1}{2}} \varepsilon^{-\frac{1}{2}} .
\end{aligned}
$$

Finally, from (2.1), (4.33), (6.14) and (6.3) it follows that

$$
\begin{aligned}
& \left\|\stackrel{\vee}{G}_{03}\left(S(k, \varepsilon) P+\varepsilon^{2} Q^{0}\right)^{-1} P\right\|_{L_{2}(\Omega) \rightarrow L_{2}(\Omega)} \leq \\
& \quad \leq\left\|g_{2}\right\|_{L_{\infty}} \varepsilon^{-\frac{1}{2}} t\|\Phi(k)\| \cdot\left\|\varepsilon D_{2}\left(S(k, \varepsilon)+\varepsilon^{2} Q^{0}\right)^{-1} P\right\|_{L_{2}(\Omega) \rightarrow L_{2}(\Omega)} \leq \\
& \quad \leq c_{1} \varepsilon^{-\frac{1}{2}} t C_{1} c_{0}^{-\frac{1}{2}}\left(c_{0} k^{2}+c_{3} \varepsilon^{2}\right)^{-\frac{1}{2}} \leq c_{1} C_{1} c_{0}^{-1} \varepsilon^{-\frac{1}{2}} .
\end{aligned}
$$

As a result, relations (8.25)

$$
\begin{aligned}
\left\|G_{03}\left(S(k, \varepsilon) P+\varepsilon^{2} Q^{0}\right)^{-1} P\right\|_{L_{2}(\Omega) \rightarrow L_{2}(\Omega)} \leq C_{8} \varepsilon^{-\frac{1}{2}} \\
\\
C_{8}=c_{1}\left(C_{7} c_{0}^{-\frac{1}{2}} c_{3}^{-\frac{1}{2}}+C_{6} C_{4} c_{3}^{-\frac{1}{2}}+C_{1} c_{0}^{-1}\right) .
\end{aligned}
$$

Next, by (6.1) and (6.12), we have

$$
\begin{aligned}
\left\|G_{3}\left(A(k, \varepsilon)+\varepsilon^{2} Q\right)^{-1}\right\|_{L_{2}(\Omega) \rightarrow L_{2}(\Omega)} & \leq \varepsilon^{\frac{1}{2}}\left\|\varepsilon D_{2}\left(A(k, \varepsilon)+\varepsilon^{2} Q\right)^{-1}\right\|_{L_{2}(\Omega) \rightarrow L_{2}(\Omega)} \leq \\
& \leq c_{0}^{-\frac{1}{2}} \varepsilon^{\frac{1}{2}}\left(c_{0} k^{2}+c_{3} \varepsilon^{2}\right)^{-\frac{1}{2}} \leq c_{0}^{-\frac{1}{2}} c_{3}^{-\frac{1}{2}} \varepsilon^{-\frac{1}{2}} .
\end{aligned}
$$




\subsection{The form $\mathcal{I}_{4}$}

It remains to study the form (8.23). It can be rewritten as

$$
\begin{aligned}
\mathcal{I}_{4}[u, v] & =\left(g_{2}^{0} \varepsilon D_{2} P u, \varepsilon D_{2} P v\right)_{L_{2}(\Omega)}-\left(g_{2} \varepsilon D_{2} P u, P \varepsilon D_{2} v\right)_{L_{2}(\Omega)}- \\
& -\left(g_{2} \varepsilon D_{2} P u, P^{\perp} \varepsilon D_{2} v\right)_{L_{2}(\Omega)} .
\end{aligned}
$$

Since $P g_{2} P=g_{2}^{0} P$, the first two terms in the right-hand side of (8.31) compensate each other. Then

$$
\mathcal{I}_{4}[u, v]=-\left(g_{2} \varepsilon D_{2} P u, P^{\perp} \varepsilon D_{2} v\right)_{L_{2}\left(\mathbb{R}^{2}\right)}=\left(G_{04} u, G_{4} v\right)_{L_{2}(\Omega)},
$$

where $G_{04}=-g_{2} \varepsilon^{\frac{3}{2}} D_{2} P, G_{4}=\varepsilon^{\frac{1}{2}} P^{\perp} D_{2}$.

By (2.1), (6.3) and (6.14), we have

$$
\begin{aligned}
\left\|G_{04}\left(S(k, \varepsilon) P+\varepsilon^{2} Q_{0}\right)^{-1} P\right\|_{L_{2}(\Omega) \rightarrow L_{2}(\Omega)} & \leq\left\|g_{2}\right\|_{L_{\infty}} \varepsilon^{\frac{1}{2}}\left\|\varepsilon D_{2}\left(S(k, \varepsilon) P+\varepsilon^{2} Q_{0}\right)^{-1} P\right\|_{L_{2}(\Omega) \rightarrow L_{2}(\Omega)} \leq \\
& \leq c_{1} \varepsilon^{\frac{1}{2}} c_{0}^{-\frac{1}{2}}\left(c_{0} k^{2}+c_{3} \varepsilon^{2}\right)^{-\frac{1}{2}} \leq c_{1} c_{0}^{-\frac{1}{2}} c_{3}^{-\frac{1}{2}} \varepsilon^{-\frac{1}{2}} .
\end{aligned}
$$

Next,

$$
\begin{aligned}
G_{4}\left(A(k, \varepsilon)+\varepsilon^{2} Q\right)^{-1} & =\varepsilon^{\frac{1}{2}} P^{\perp} D_{2}\left(A(k, \varepsilon)+\varepsilon^{2} Q\right)^{-1}= \\
& =\varepsilon^{\frac{1}{2}} P^{\perp}\left[D_{2},\left(A(k, \varepsilon)+\varepsilon^{2} Q\right)^{-1}\right]+\varepsilon^{-\frac{1}{2}} P^{\perp}\left(A(k, \varepsilon)+\varepsilon^{2} Q\right)^{-1} \varepsilon D_{2} .
\end{aligned}
$$

The first term on the right can be estimated with the help of (7.5), and the second one is estimated by using (6.12) and (6.17). As a result, we obtain that

$$
\begin{aligned}
\left\|G_{4}\left(A(k, \varepsilon)+\varepsilon^{2} Q\right)^{-1}\right\|_{L_{2}(\Omega) \rightarrow L_{2}(\Omega)} & \leq \varepsilon^{\frac{1}{2}} C_{5}\left(c_{0} k^{2}+c_{3} \varepsilon^{2}\right)^{-\frac{1}{2}}+\varepsilon^{-\frac{1}{2}} C_{3} c_{0}^{-\frac{1}{2}} \leq \\
& \leq\left(C_{5} c_{3}^{-\frac{1}{2}}+C_{3} c_{0}^{-\frac{1}{2}}\right) \varepsilon^{-\frac{1}{2}} .
\end{aligned}
$$

\subsection{Representation of the form $\mathcal{I}$}

Now we summarize the study of the form (8.4). By (8.4), (8.8), 8.12), (8.15), 8.18), (8.21), (8.24), (8.32), this form is represented as

$$
\begin{aligned}
\mathcal{I}[u, v] & =\left(G_{01}^{\prime} u, G_{1}^{\prime} v\right)_{L_{2}(\Omega)}+\left(G_{01}^{\prime \prime} u, G_{1}^{\prime \prime} v\right)_{L_{2}(\Omega)}+ \\
& +\left(G_{02} u, G_{2} v\right)_{L_{2}(\Omega)}+\left(G_{03} u, G_{3} v\right)_{L_{2}(\Omega)}+\left(G_{04} u, G_{4} v\right)_{L_{2}(\Omega)} .
\end{aligned}
$$

We put $\mathfrak{G}=L_{2}\left(\Omega ; \mathbb{C}^{5}\right)=\left(L_{2}(\Omega)\right)^{5}$ and introduce the operators

$$
\begin{aligned}
G_{0} & : L_{2}(\Omega) \rightarrow \mathfrak{G}, G_{0}=\operatorname{col}\left\{G_{01}^{\prime}, G_{01}^{\prime \prime}, G_{02}, G_{03}, G_{04}\right\}, \\
G & : L_{2}(\Omega) \rightarrow \mathfrak{G}, G=\operatorname{col}\left\{G_{1}^{\prime}, G_{1}^{\prime \prime}, G_{2}, G_{3}, G_{4}\right\}, \\
\operatorname{Dom} G_{0} & =\operatorname{Dom} G=\left\{u \in L_{2}(\Omega): D_{2} u \in L_{2}(\Omega)\right\} .
\end{aligned}
$$

The operators $G_{0}$ and $G$ are densely defined, and we have

$$
\mathfrak{d}=\widetilde{H}^{1}(\Omega) \subset \operatorname{Dom} G_{0}=\operatorname{Dom} G,
$$

and

$$
\mathcal{I}[u, v]=\left(G_{0} u, G v\right)_{\mathfrak{G}}, \quad u, v \in \mathfrak{d} .
$$

Relations (8.14), (8.17), (8.20), (8.30), (8.34) show that the operator $G\left(A(k, \varepsilon)+\varepsilon^{2} Q\right)^{-1}$ is bounded and

$$
\left\|G\left(A(k, \varepsilon)+\varepsilon^{2} Q\right)^{-1}\right\|_{L_{2}(\Omega) \rightarrow \mathfrak{G}} \leq C_{9} \varepsilon^{-\frac{1}{2}},
$$


where

$$
C_{9}^{2}=C_{3}^{2} c_{3}^{-1}+c_{0}^{-\frac{1}{2}} c_{3}^{-\frac{3}{2}}+c_{0}^{-\frac{3}{2}} c_{3}^{-\frac{1}{2}}+c_{0}^{-1} c_{3}^{-1}+\left(C_{5} c_{3}^{-\frac{1}{2}}+C_{3} c_{0}^{-\frac{1}{2}}\right)^{2} .
$$

Inequalities (8.13), (8.16), (8.19), (8.29) and (8.33) imply that the operator $G_{0}\left(S(k, \varepsilon) P+\varepsilon^{2} Q^{0}\right)^{-1} P$ is bounded and

$$
\left\|G_{0}\left(S(k, \varepsilon) P+\varepsilon^{2} Q^{0}\right)^{-1} P\right\|_{L_{2}(\Omega) \rightarrow \mathfrak{G}} \leq C_{10} \varepsilon^{-\frac{1}{2}},
$$

where

$$
C_{10}^{2}=c_{4}^{2} c_{3}^{-2}+C_{1}^{2} c_{4}^{2} c_{0}^{-\frac{1}{2}} c_{3}^{-\frac{3}{2}}+C_{2}^{2} c_{0}^{-\frac{3}{2}} c_{3}^{-\frac{1}{2}}+C_{8}^{2}+c_{1}^{2} c_{0}^{-1} c_{3}^{-1} .
$$

Thus, we have checked that in our case condition $5^{\circ}$ of Lemma 14 is satisfied.

Applying Lemma 14, we obtain representation of the form (8.3) for the operator (6.23):

$$
\begin{aligned}
\mathcal{J}(k, \varepsilon) & =\left(A(k, \varepsilon)+\varepsilon^{2} Q\right)^{-1} P-F(k)\left(S(k, \varepsilon)+\varepsilon^{2} Q^{0}\right)^{-1} P= \\
& =\left(G\left(A(k, \varepsilon)+\varepsilon^{2} Q\right)^{-1}\right)^{*}\left(G_{0}\left(S(k, \varepsilon) P+\varepsilon^{2} Q^{0}\right)^{-1} P\right) .
\end{aligned}
$$

Then relations (8.36), (8.38) and (8.40) imply that

$$
\|\mathcal{J}(k, \varepsilon)\|_{L_{2}(\Omega) \rightarrow L_{2}(\Omega)} \leq C_{9} C_{10} \varepsilon^{-1}, \quad|k| \leq t_{0}, \quad 0<\varepsilon \leq 1 .
$$

This proves estimate (6.24) with $\widehat{C}=C_{9} C_{10}$.

This completes the proof of Proposition 9, and, with it, of Theorem 4 Also, this completes the proof of Theorem 3 (see Subsection 5.2) and, thereby (see Subsection 3.2), that of Theorem 1.

\section{Application to the Schrödinger operator with singular peri- odic potential}

Homogenization problem for the Schrödinger operator $\mathcal{H}_{\varepsilon}=-\operatorname{div} g(\mathbf{x} / \varepsilon) \nabla+\varepsilon^{-2} V(\mathbf{x} / \varepsilon)$ in $L_{2}\left(\mathbb{R}^{d}\right)$ with coefficients periodic in all directions was studied by a spectral method in Ch. 6, $\S 1$ of [BSu1], $\S 11$ of [BSu3], $\S 18$ of [BSu4. Investigation was based on a special factorization for the Schrödinger operator (see [KiSi] and $\S 4$ of [BSu5]).

Here we apply Theorem 1 to homogenization problem for the Schrödinger operator of the form

$$
H_{\varepsilon}=D_{1} \widetilde{g}_{1}\left(\varepsilon^{-1} x_{1}, x_{2}\right) D_{1}+D_{2} \widetilde{g}_{2}\left(\varepsilon^{-1} x_{1}, x_{2}\right) D_{2}+\varepsilon^{-2} V\left(\varepsilon^{-1} x_{1}, x_{2}\right)
$$

with metric and potential periodic in $x_{1}$. The operator $H_{\varepsilon}$ contains a large factor $\varepsilon^{-2}$ standing at $V\left(\varepsilon^{-1} x_{1}, x_{2}\right)$; in this sense the potential is singular. Under some restrictions on the coefficients (potential $V$ must admit a certain representation), we obtain approximation for the resolvent $\left(H_{\varepsilon}+\lambda I\right)^{-1}$ in the operator $L_{2}$-norm with sharp order error estimate.

\subsection{Conditions on coefficients}

Let $\widetilde{g}_{1}(\mathbf{x}), \widetilde{g}_{2}(\mathbf{x})$ be measurable functions in $\mathbb{R}^{2}$ satisfying the same conditions as $g_{1}, g_{2}$. Namely, let $\widetilde{g}_{j}$, $j=1,2$, be periodic in $x_{1}$ with period 1 , uniformly bounded and positive definite:

$$
\widetilde{c}_{0} \leq \widetilde{g}_{j}(\mathbf{x}) \leq \widetilde{c}_{1}<\infty, \quad j=1,2, \text { a. e. } \mathbf{x} \in \mathbb{R}^{2} .
$$

Also, it is assumed that $\widetilde{g}_{j}$ are Lipschitz functions with respect to $x_{2}$ :

$$
\underset{\mathbf{x} \in \mathbb{R}^{2}}{\operatorname{ess} \sup }\left|\partial_{2} \widetilde{g}_{j}(\mathbf{x})\right| \leq \widetilde{c}_{2}<\infty, \quad j=1,2 .
$$

Next, let $\omega(\mathbf{x})$ be a measurable function in $\mathbb{R}^{2}$ such that

$$
0<\omega_{0} \leq \omega(\mathbf{x}) \leq \omega_{1}<\infty, \quad \text { a. e. } \mathbf{x} \in \mathbb{R}^{2} .
$$


Assume that $\omega(\mathbf{x})$ is 1-periodic in $x_{1}$ and Lipschitz class in $x_{2}$ :

$$
\underset{\mathbf{x} \in \mathbb{R}^{2}}{\operatorname{essup}}\left|\partial_{2} \omega(\mathbf{x})\right| \leq \widetilde{c}_{3}<\infty
$$

We put

$$
\begin{aligned}
V(\mathbf{x}) & =-\frac{D_{1} \widetilde{g}_{1}(\mathbf{x}) D_{1} \omega(\mathbf{x})}{\omega(\mathbf{x})}, \\
V_{2}(\mathbf{x}) & =-\frac{D_{2} \widetilde{g}_{2}(\mathbf{x}) D_{2} \omega(\mathbf{x})}{\omega(\mathbf{x})} .
\end{aligned}
$$

Additional assumptions on $\widetilde{g}_{1}, \widetilde{g}_{2}$ and $\omega$ are formulated in terms of the functions (9.6), (9.7). Namely, assume that $V_{2}$ is uniformly bounded:

$$
\left|V_{2}(\mathbf{x})\right| \leq \widetilde{c}_{4}, \quad \text { a. e. } \mathbf{x} \in \mathbb{R}^{2},
$$

and Lipschitz class with respect to $x_{2}$ :

$$
\underset{\mathbf{x} \in \mathbb{R}^{2}}{\operatorname{ess} \sup _{2}}\left|\partial_{2} V_{2}(\mathbf{x})\right| \leq \widetilde{c}_{5}<\infty
$$

Assume that $V\left(\cdot, x_{2}\right) \in L_{1}(0,1)$ for a. e. $x_{2} \in \mathbb{R}$ and

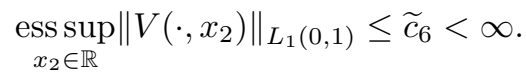

Using (9.2), (9.4), (9.6) and (9.10), it is easily seen that $\omega(\mathbf{x})$ is Lipschitz in $x_{1}$ :

$$
\underset{\mathbf{x} \in \mathbb{R}^{2}}{\operatorname{ess} \sup _{1}}\left|\partial_{1} \omega(\mathbf{x})\right| \leq \widetilde{c}_{7}=\omega_{1} \widetilde{c}_{6}\left(\widetilde{c}_{0}^{-1}+\widetilde{c}_{1} \widetilde{c}_{0}^{-2}\right)<\infty
$$

Then, by (9.4), (9.5) and (9.11), functions $\omega$ and $\omega^{-1}$ are multipliers in the Sobolev class $H^{1}\left(\mathbb{R}^{2}\right)$.

\subsection{Operators $\widetilde{A}_{\varepsilon}, A_{\varepsilon}$ and $H_{\varepsilon}$}

For any measurable function $\varphi\left(x_{1}, x_{2}\right)$ which is 1 -periodic in $x_{1}$, we use the following notation

$$
\varphi^{\varepsilon}\left(x_{1}, x_{2}\right)=\varphi\left(\varepsilon^{-1} x_{1}, x_{2}\right) .
$$

In $L_{2}\left(\mathbb{R}^{2}\right)$, consider the operator $\widetilde{A}_{\varepsilon}$ formally given by the expression

$$
\widetilde{A}_{\varepsilon}=\left(\omega^{\varepsilon}\right)^{-1} D_{1} \widetilde{g}_{1}^{\varepsilon}\left(\omega^{\varepsilon}\right)^{2} D_{1}\left(\omega^{\varepsilon}\right)^{-1}+\left(\omega^{\varepsilon}\right)^{-1} D_{2} \widetilde{g}_{2}^{\varepsilon}\left(\omega^{\varepsilon}\right)^{2} D_{2}\left(\omega^{\varepsilon}\right)^{-1}
$$

Obviously,

$$
\widetilde{A}_{\varepsilon}=\left(\omega^{\varepsilon}\right)^{-1} A_{\varepsilon}\left(\omega^{\varepsilon}\right)^{-1}
$$

where $A_{\varepsilon}$ is given by

$$
A_{\varepsilon}=D_{1} g_{1}^{\varepsilon} D_{1}+D_{2} g_{2}^{\varepsilon} D_{2}
$$

(for precise definition of $A_{\varepsilon}$, see Section 2) with the coefficients

$$
g_{j}(\mathbf{x})=\widetilde{g}_{j}(\mathbf{x}) \omega^{2}(\mathbf{x}), \quad j=1,2 .
$$

Due to conditions (9.2) and periodicity of $\widetilde{g}_{j}, \omega$ in $x_{1}$, coefficients (9.15) satisfy the required conditions (2.1)-(2.3).

The precise definition of $\widetilde{A}_{\varepsilon}$ is given in terms of the quadratic form

$$
\widetilde{a}_{\varepsilon}[u, u]=\int_{\mathbb{R}^{2}}\left(g_{1}^{\varepsilon}\left|D_{1}\left(\omega^{\varepsilon}\right)^{-1} u\right|^{2}+g_{2}^{\varepsilon}\left|D_{2}\left(\omega^{\varepsilon}\right)^{-1} u\right|^{2}\right) d \mathbf{x}, \quad u \in H^{1}\left(\mathbb{R}^{2}\right) .
$$


By direct calculations, it is easy to check the following identity:

$$
\widetilde{a}_{\varepsilon}[u, u]=\int_{\mathbb{R}^{2}}\left(\widetilde{g}_{1}^{\varepsilon}\left|D_{1} u\right|^{2}+\widetilde{g}_{2}^{\varepsilon}\left|D_{2} u\right|^{2}+\varepsilon^{-2} V^{\varepsilon}|u|^{2}+V_{2}^{\varepsilon}|u|^{2}\right) d \mathbf{x}, \quad u \in H^{1}\left(\mathbb{R}^{2}\right) .
$$

Now, we introduce the Schrödinger operator $H_{\varepsilon}$ formally given by (9.1). The precise definition of $H_{\varepsilon}$ is given in terms of the quadratic form

$$
h_{\varepsilon}[u, u]=\int_{\mathbb{R}^{2}}\left(\widetilde{g}_{1}^{\varepsilon}\left|D_{1} u\right|^{2}+\widetilde{g}_{2}^{\varepsilon}\left|D_{2} u\right|^{2}+\varepsilon^{-2} V^{\varepsilon}|u|^{2}\right) d \mathbf{x}, \quad u \in H^{1}\left(\mathbb{R}^{2}\right) .
$$

Note that conditions (9.2) and (9.10) together with periodicity of coefficients in $x_{1}$ ensure that the form (9.18) is closed and lower semibounded on domain $H^{1}\left(\mathbb{R}^{2}\right)$ (the same is true for the form in the right-hand side of (9.17)).

Relations (9.17) and (9.18) mean that

$$
H_{\varepsilon}=\widetilde{A}_{\varepsilon}-V_{2}^{\varepsilon}
$$

\subsection{Homogenization for the Schrödinger operator $H_{\varepsilon}$}

From (9.19) and (9.13) it follows that

$$
H_{\varepsilon}+\lambda I=\widetilde{A}_{\varepsilon}-V_{2}^{\varepsilon}+\lambda I=\left(\omega^{\varepsilon}\right)^{-1}\left(A_{\varepsilon}+Q_{\lambda}^{\varepsilon}\right)\left(\omega^{\varepsilon}\right)^{-1}, \quad \lambda \in \mathbb{R},
$$

where

$$
Q_{\lambda}(\mathbf{x})=\left(\lambda-V_{2}(\mathbf{x})\right) \omega^{2}(\mathbf{x}) .
$$

By (9.4) and (9.8), we have $Q_{\lambda}(\mathbf{x}) \geq \lambda \omega_{0}^{2}-\omega_{1}^{2} \widetilde{c}_{4}$ for a. e. $\mathbf{x} \in \mathbb{R}^{2}$. Assume that $\lambda$ is sufficiently large so that

$$
\lambda \omega_{0}^{2}-\omega_{1}^{2} \widetilde{c}_{4}=: \widetilde{c}_{\lambda}>0 .
$$

Due to (9.4), (9.5), (9.8), (9.9), (9.22) and periodicity conditions, the function (9.21) satisfies the same conditions as $Q$ (see (2.5)-(2.7)). We put

$$
Q_{\lambda}^{0}\left(x_{2}\right)=\int_{0}^{1} Q_{\lambda}\left(x_{1}, x_{2}\right) d x_{1} .
$$

Let $A^{0}$ be the effective operator corresponding to $A_{\varepsilon}$, i. e.,

$$
A^{0}=D_{1} g_{1}^{0}\left(x_{2}\right) D_{1}+D_{2} g_{2}^{0}\left(x_{2}\right) D_{2},
$$

where the coefficients $g_{1}^{0}, g_{2}^{0}$ are defined according to (2.10), (2.11) in terms of the coefficients (9.15).

By Theorem 1, we have

$$
\left\|\left(A_{\varepsilon}+Q_{\lambda}^{\varepsilon}\right)^{-1}-\left(A^{0}+Q_{\lambda}^{0}\right)^{-1}\right\|_{L_{2}\left(\mathbb{R}^{2}\right) \rightarrow L_{2}\left(\mathbb{R}^{2}\right)} \leq \widetilde{C}_{\lambda} \varepsilon .
$$

Here the constant $\widetilde{C}_{\lambda}$ depends only on $\widetilde{c}_{0}, \widetilde{c}_{1}, \widetilde{c}_{2}, \omega_{0}, \omega_{1}, \widetilde{c}_{3}, \widetilde{c}_{4}, \widetilde{c}_{5}$ and $\lambda$. Multiplying operators under the norm sign in (9.25) by $\omega^{\varepsilon}$ from both sides and using identity (9.20), we arrive at the following result.

Theorem 15 Let $\widetilde{g}_{1}, \widetilde{g}_{2}$, $\omega$ be measurable functions in $\mathbb{R}^{2}$, periodic in $x_{1}$ with period 1 and satisfying conditions (9.2)-(9.5). Suppose that the functions $V, V_{2}$ defined by (9.6), (9.7) satisfy conditions (9.8)(9.10). Let $H_{\varepsilon}$ be the operator in $L_{2}\left(\mathbb{R}^{2}\right)$ corresponding to the quadratic form (9.18). Let $A_{\varepsilon}$ be the operator (9.14) with the coefficients (9.15), and let $A^{0}$ be the corresponding effective operator (9.24) with coefficients defined according to (2.10), (2.11). Let $Q_{\lambda}$ be defined by (9.21), and let restriction (9.22) be satisfied. Let $Q_{\lambda}^{0}$ be given by (9.23). Then we have

$$
\left\|\left(H_{\varepsilon}+\lambda I\right)^{-1}-\omega^{\varepsilon}\left(A^{0}+Q_{\lambda}^{0}\right)^{-1} \omega^{\varepsilon}\right\|_{L_{2}\left(\mathbb{R}^{2}\right) \rightarrow L_{2}\left(\mathbb{R}^{2}\right)} \leq C_{\lambda} \varepsilon, \quad 0<\varepsilon \leq 1 .
$$

Here the constant $C_{\lambda}=\omega_{1}^{2} \widetilde{C}_{\lambda}$ depends only on $\widetilde{c}_{0}, \widetilde{c}_{1}, \widetilde{c}_{2}, \omega_{0}, \omega_{1}, \widetilde{c}_{3}, \widetilde{c}_{4}, \widetilde{c}_{5}$ and $\lambda$. 
Note that the approximate operator in (9.26) contains rapidly oscillating factors $\omega^{\varepsilon}$ from both sides of the operator $\left(A^{0}+Q_{\lambda}^{0}\right)^{-1}$, but the inverse is taken only for the differential operator $A^{0}+Q_{\lambda}^{0}$ with constant coefficients. Such kind of results is typical also for the Schrödinger operator with coefficients periodic in all directions (see [BSu1, Ch. 6, §1).

\subsection{About representation (9.6)}

A natural question is the following. If the initial object is the Schrödinger operator (9.1) with given coefficients $\widetilde{g}_{1}, \widetilde{g}_{2}, V$, then is it possible to find $\omega$ such that representation (9.6) is true and conditions of Theorem 18 are satisfied?

For a.e. $x_{2} \in \mathbb{R}$, consider the Schrödinger operator

$$
H\left(x_{2}\right)=D_{1} \widetilde{g}_{1}\left(x_{1}, x_{2}\right) D_{1}+V\left(x_{1}, x_{2}\right),
$$

acting in $L_{2}(\mathbb{R})$ and depending on parameter $x_{2}$. We assume now that $\widetilde{g}_{1}$ and $V$ are 1-periodic in $x_{1}$ and satisfy (9.2) and (9.10). Assume also that the bottom of the spectrum of the operator (9.27) coincides with point $\lambda=0$ :

$$
\inf \operatorname{spec} H\left(x_{2}\right)=0 .
$$

If initially condition (9.28) was not satisfied, it is possible to ensure this condition replacing $V\left(x_{1}, x_{2}\right)$ by $V\left(x_{1}, x_{2}\right)-\lambda\left(x_{2}\right)$, where $\lambda\left(x_{2}\right)=\inf \operatorname{spec} H\left(x_{2}\right)$.

If condition (9.28) is satisfied, there exists a positive periodic (in $\left.x_{1}\right)$ solution $\omega\left(x_{1}, x_{2}\right)$ of the equation

$$
D_{1} \widetilde{g}_{1}\left(x_{1}, x_{2}\right) D_{1} \omega\left(x_{1}, x_{2}\right)+V\left(x_{1}, x_{2}\right) \omega\left(x_{1}, x_{2}\right)=0 .
$$

This solution can be fixed by the condition

$$
\int_{0}^{1} \omega^{2}\left(x_{1}, x_{2}\right) d x_{1}=1
$$

Moreover, this solution satisfies (9.4), where $\omega_{0}, \omega_{1}$ are controlled in terms of $\widetilde{c}_{0}, \widetilde{c}_{1}, \widetilde{c}_{6}$. Concerning the properties of solution $\omega$, see [KiSi] and $\S 4$ of [BSu5]. Thus, by (9.29), $V$ satisfies representation (9.6).

Next, it is possible to ensure conditions (9.5), (9.8) and (9.9) imposing some smoothness assumptions on coefficients $\widetilde{g}_{j}, V$ with respect to $x_{2}$. It suffices to assume that derivatives $\partial_{2}^{l} \widetilde{g}_{1}, l=1,2,3$, are uniformly bounded, derivatives $\partial_{2}^{l} \widetilde{g}_{2}, l=1,2$, are uniformly bounded and that the norms $\left\|\partial_{2}^{l} V\left(\cdot, x_{2}\right)\right\|_{L_{1}(0,1)}$, $l=1,2,3$, are uniformly bounded.

Under the above assumptions, all conditions of Theorem 18 are satisfied.

\section{Generalization of the main results for the case of arbitrary dimension}

\subsection{Generalization of Theorem 1}

One can study an analog of the operator $A_{\varepsilon}$ in $L_{2}\left(\mathbb{R}^{d}\right)$ with $d=d_{1}+d_{2} \geq 2, d_{1}, d_{2} \geq 1$. We use the notation $\mathbf{x}=\left(\mathbf{x}^{\prime}, \mathbf{x}^{\prime \prime}\right), \mathbf{x}^{\prime} \in \mathbb{R}^{d_{1}}, \mathbf{x}^{\prime \prime} \in \mathbb{R}^{d_{2}}, \mathbf{D}_{\mathbf{x}^{\prime}}=-i \nabla_{\mathbf{x}^{\prime}}, \mathbf{D}_{\mathbf{x}^{\prime}}^{*}=-i \operatorname{div}_{\mathbf{x}^{\prime}}$, and similarly for $\mathbf{D}_{\mathbf{x}^{\prime \prime}}, \mathbf{D}_{\mathbf{x}^{\prime \prime}}^{*}$. Let $g_{1}(\mathbf{x})=g_{1}\left(\mathbf{x}^{\prime}, \mathbf{x}^{\prime \prime}\right)$ be a $\left(d_{1} \times d_{1}\right)$-matrix-valued measurable function and $g_{2}(\mathbf{x})=g_{2}\left(\mathbf{x}^{\prime}, \mathbf{x}^{\prime \prime}\right)$ be a $\left(d_{2} \times d_{2}\right)$-matrix-valued measurable function in $\mathbb{R}^{d}$. Assume that $g_{1}(\mathbf{x}), g_{2}(\mathbf{x})$ are bounded and positive definite:

$$
c_{0} \mathbf{1}_{d_{1}} \leq g_{1}(\mathbf{x}) \leq c_{1} \mathbf{1}_{d_{1}}, \quad c_{0} \mathbf{1}_{d_{2}} \leq g_{2}(\mathbf{x}) \leq c_{1} \mathbf{1}_{d_{2}}, \quad 0<c_{0} \leq c_{1}<\infty, \text { a. e. } \mathbf{x} \in \mathbb{R}^{d} .
$$

Assume also that $g_{1}(\mathbf{x}), g_{2}(\mathbf{x})$ are periodic in $\mathbf{x}^{\prime}$ with respect to some lattice $\Gamma \subset \mathbb{R}^{d_{1}}$, and that $g_{1}, g_{2}$ are Lipschitz class with respect to $\mathbf{x}^{\prime \prime}$ :

$$
\underset{\mathbf{x} \in \mathbb{R}^{d}}{\operatorname{ess} \sup ^{\prime}}\left|\mathbf{D}_{\mathbf{x}^{\prime \prime}} g_{j}(\mathbf{x})\right| \leq c_{2}<\infty, \quad j=1,2 .
$$


In $L_{2}\left(\mathbb{R}^{d}\right)$, consider the operator $A_{\varepsilon}$ formally given by the differential expression

$$
A_{\varepsilon}=\mathbf{D}_{\mathbf{x}^{\prime}}^{*} g_{1}\left(\varepsilon^{-1} \mathbf{x}^{\prime}, \mathbf{x}^{\prime \prime}\right) \mathbf{D}_{\mathbf{x}^{\prime}}+\mathbf{D}_{\mathbf{x}^{\prime \prime}}^{*} g_{1}\left(\varepsilon^{-1} \mathbf{x}^{\prime}, \mathbf{x}^{\prime \prime}\right) \mathbf{D}_{\mathbf{x}^{\prime \prime}}, \quad \varepsilon>0 .
$$

Precisely, $A_{\varepsilon}$ is a selfadjoint operator in $L_{2}\left(\mathbb{R}^{d}\right)$ corresponding to the quadratic form

$$
a_{\varepsilon}[u, u]=\int_{\mathbb{R}^{d}}\left(\left\langle g_{1}\left(\varepsilon^{-1} \mathbf{x}^{\prime}, \mathbf{x}^{\prime \prime}\right) \mathbf{D}_{\mathbf{x}^{\prime}} u, \mathbf{D}_{\mathbf{x}^{\prime}} u\right\rangle_{\mathbb{C}^{d_{1}}}+\left\langle g_{2}\left(\varepsilon^{-1} \mathbf{x}^{\prime}, \mathbf{x}^{\prime \prime}\right) \mathbf{D}_{\mathbf{x}^{\prime \prime}} u, \mathbf{D}_{\mathbf{x}^{\prime \prime}} u\right\rangle_{\mathbb{C}^{d_{2}}}\right) d \mathbf{x}, \quad u \in H^{1}\left(\mathbb{R}^{d}\right) .
$$

Let $Q(\mathbf{x})=Q\left(\mathbf{x}^{\prime}, \mathbf{x}^{\prime \prime}\right)$ be a real-valued measurable function in $\mathbb{R}^{d}$ such that

$$
0<c_{3} \leq Q(\mathbf{x}) \leq c_{4}<\infty, \text { a. e. } \mathbf{x} \in \mathbb{R}^{d} .
$$

Assume that $Q(\mathbf{x})$ is $\Gamma$-periodic in $\mathbf{x}^{\prime}$, and that

$$
\underset{\mathbf{x} \in \mathbb{R}^{d}}{\operatorname{ess} \sup }\left|\mathbf{D}_{\mathbf{x}^{\prime \prime}} Q(\mathbf{x})\right| \leq c_{5}<\infty .
$$

We put

$$
Q^{\varepsilon}\left(\mathbf{x}^{\prime}, \mathbf{x}^{\prime \prime}\right)=Q\left(\varepsilon^{-1} \mathbf{x}^{\prime}, \mathbf{x}^{\prime \prime}\right) .
$$

For the generalized resolvent $\left(A_{\varepsilon}+Q^{\varepsilon}\right)^{-1}$, the analog of Theorem 11 is true. The effective operator $A^{0}$ corresponds to the differential expression

$$
A^{0}=\mathbf{D}_{\mathbf{x}^{\prime}}^{*} g_{1}^{0}\left(\mathbf{x}^{\prime \prime}\right) \mathbf{D}_{\mathbf{x}^{\prime}}+\mathbf{D}_{\mathbf{x}^{\prime \prime}}^{*} g_{2}^{0}\left(\mathbf{x}^{\prime \prime}\right) \mathbf{D}_{\mathbf{x}^{\prime \prime}} .
$$

Here the effective coefficients $g_{1}^{0}\left(\mathbf{x}^{\prime \prime}\right), g_{2}^{0}\left(\mathbf{x}^{\prime \prime}\right)$ are defined as follows. Let $\Omega^{\prime}$ be an elementary cell of the lattice $\Gamma \subset \mathbb{R}^{d_{1}}$. Then

$$
g_{2}^{0}\left(\mathbf{x}^{\prime \prime}\right)=\mid \Omega^{\prime-1} \int_{\Omega^{\prime}} g_{2}\left(\mathbf{x}^{\prime}, \mathbf{x}^{\prime \prime}\right) d \mathbf{x}^{\prime} .
$$

The matrix $g_{1}^{0}\left(\mathbf{x}^{\prime \prime}\right)$ is the effective matrix corresponding to the elliptic operator $\mathbf{D}_{\mathbf{x}^{\prime}}^{*} g_{1}\left(\mathbf{x}^{\prime}, \mathbf{x}^{\prime \prime}\right) \mathbf{D}_{\mathbf{x}^{\prime}}$ in $L_{2}\left(\mathbb{R}^{d_{1}}\right)$. Recall the definition of $g_{1}^{0}$. Let $\mathbf{e}_{1}, \ldots, \mathbf{e}_{d_{1}}$ be the standard orthonormal basis in $\mathbb{C}^{d_{1}}$, and let $\Phi_{j}(\mathbf{x})$ be a $\Gamma$-periodic (in $\mathbf{x}^{\prime}$ ) solution of the equation

$$
\operatorname{div}_{\mathbf{x}^{\prime}} g_{1}\left(\mathbf{x}^{\prime}, \mathbf{x}^{\prime \prime}\right)\left(\nabla_{\mathbf{x}^{\prime}} \Phi_{j}\left(\mathbf{x}^{\prime}, \mathbf{x}^{\prime \prime}\right)+\mathbf{e}_{j}\right)=0,
$$

$j=1, \ldots, d_{1}$. Here $\mathbf{x}^{\prime \prime}$ is a parameter. Then $g_{1}^{0}\left(\mathbf{x}^{\prime \prime}\right)$ is a $\left(d_{1} \times d_{1}\right)$-matrix with the columns

$$
\mathbf{g}_{j}\left(\mathbf{x}^{\prime \prime}\right)=\mid \Omega^{\prime-1} \int_{\Omega^{\prime}} g_{1}\left(\mathbf{x}^{\prime}, \mathbf{x}^{\prime \prime}\right)\left(\nabla_{\mathbf{x}^{\prime}} \Phi_{j}\left(\mathbf{x}^{\prime}, \mathbf{x}^{\prime \prime}\right)+\mathbf{e}_{j}\right) d \mathbf{x}^{\prime}, \quad j=1, \ldots, d_{1} .
$$

Note that for $d_{1}=1$ relation (10.10) is equivalent to (2.10).

Finally, let

$$
Q^{0}\left(\mathbf{x}^{\prime \prime}\right)=\mid \Omega^{\prime-1} \int_{\Omega^{\prime}} Q\left(\mathbf{x}^{\prime}, \mathbf{x}^{\prime \prime}\right) d \mathbf{x}^{\prime}
$$

The following result can be proved by analogy with the proof of Theorem 1,

Theorem 16 Let $d=d_{1}+d_{2} \geq 2, d_{1}, d_{2} \geq 1$. Let $g_{1}(\mathbf{x})$ and $g_{2}(\mathbf{x})$ be measurable matrix-valued functions in $\mathbb{R}^{d}$ (of sizes $d_{1} \times d_{1}$ and $d_{2} \times d_{2}$ correspondingly). Suppose that $g_{1}, g_{2}$ are $\Gamma$-periodic in $\mathbf{x}^{\prime}$ and satisfy conditions (10.1), (10.2). Let $A_{\varepsilon}$ be the operator in $L_{2}\left(\mathbb{R}^{d}\right)$ corresponding to the form (10.4). Let $Q(\mathbf{x})$ be a measurable function in $\mathbb{R}^{d}$, $\Gamma$-periodic in $\mathbf{x}^{\prime}$ and satisfying conditions (10.5), (10.6). Let $Q^{\varepsilon}(\mathbf{x})$ be defined by (10.7). Let $A^{0}$ be the operator (10.8), where the coefficients $g_{1}^{0}, g_{2}^{0}$ are defined by (10.10) and (10.9) respectively. Let $Q^{0}$ be defined by (10.11). Then we have

$$
\left\|\left(A_{\varepsilon}+Q^{\varepsilon}\right)^{-1}-\left(A^{0}+Q^{0}\right)^{-1}\right\|_{L_{2}\left(\mathbb{R}^{d}\right) \rightarrow L_{2}\left(\mathbb{R}^{d}\right)} \leq C \varepsilon, \quad 0<\varepsilon \leq 1,
$$

where the constant $C$ depends only on $c_{j}, j=0, \ldots, 5$, and on parameters of the lattice $\Gamma$. 


\subsection{Generalization of Theorem 18}

Let $\widetilde{g}_{j}(\mathbf{x}), j=1,2$, be measurable matrix-valued functions in $\mathbb{R}^{d}$ satisfying the same conditions as $g_{j}$. Namely, we assume that $\widetilde{g}_{j}, j=1,2$, are $\Gamma$-periodic in $\mathbf{x}^{\prime}$, bounded and positive definite:

$$
\widetilde{c}_{0} \mathbf{1}_{d_{1}} \leq \widetilde{g}_{1}(\mathbf{x}) \leq \widetilde{c}_{1} \mathbf{1}_{d_{1}}, \quad \widetilde{c}_{0} \mathbf{1}_{d_{2}} \leq \widetilde{g}_{2}(\mathbf{x}) \leq \widetilde{c}_{1} \mathbf{1}_{d_{2}}, \quad 0<c_{0} \leq c_{1}<\infty, \text { a. e. } \mathbf{x} \in \mathbb{R}^{d} .
$$

Assume also that $\widetilde{g}_{1}(\mathbf{x}), \widetilde{g}_{2}(\mathbf{x})$ are Lipschitz with respect to $\mathbf{x}^{\prime \prime}$ :

$$
\underset{\mathbf{x} \in \mathbb{R}^{d}}{\operatorname{ess} \sup ^{\prime}}\left|\mathbf{D}_{\mathbf{x}^{\prime \prime}} \widetilde{g}_{j}(\mathbf{x})\right| \leq c_{2}<\infty, \quad j=1,2 .
$$

Next, let $\omega(\mathbf{x})$ be a measurable function in $\mathbb{R}^{d}$ such that

$$
0<\omega_{0} \leq \omega(\mathbf{x}) \leq \omega_{1}<\infty, \quad \text { a. e. } \mathbf{x} \in \mathbb{R}^{d} .
$$

Assume that $\omega(\mathbf{x})$ is $\Gamma$-periodic in $\mathbf{x}^{\prime}$ and Lipschitz class in $\mathbf{x}^{\prime \prime}$ :

$$
\underset{\mathbf{x} \in \mathbb{R}^{2}}{\operatorname{esssup}}\left|\mathbf{D}_{\mathbf{x}^{\prime \prime}} \omega(\mathbf{x})\right| \leq \widetilde{c}_{3}<\infty .
$$

We put

$$
\begin{aligned}
V(\mathbf{x}) & =-\frac{\mathbf{D}_{\mathbf{x}^{\prime}}^{*} \widetilde{g}_{1}(\mathbf{x}) \mathbf{D}_{\mathbf{x}^{\prime}} \omega(\mathbf{x})}{\omega(\mathbf{x})}, \\
V_{2}(\mathbf{x}) & =-\frac{\mathbf{D}_{\mathbf{x}^{\prime \prime}}^{*} \widetilde{g}_{2}(\mathbf{x}) \mathbf{D}_{\mathbf{x}^{\prime \prime}} \omega(\mathbf{x})}{\omega(\mathbf{x})} .
\end{aligned}
$$

Additional assumptions on $\widetilde{g}_{1}, \widetilde{g}_{2}$ and $\omega$ are formulated in terms of the functions (10.17), (10.18). Namely, assume that $V_{2}$ is uniformly bounded:

$$
\left|V_{2}(\mathbf{x})\right| \leq \widetilde{c}_{4}, \quad \text { a. e. } \mathbf{x} \in \mathbb{R}^{d},
$$

and Lipschitz class with respect to $\mathbf{x}^{\prime \prime}$ :

$$
\underset{\mathbf{x} \in \mathbb{R}^{2}}{\operatorname{ess} \sup ^{2}}\left|\mathbf{D}_{\mathbf{x}^{\prime \prime}} V_{2}(\mathbf{x})\right| \leq \widetilde{c}_{5}<\infty .
$$

Assume that $V\left(\cdot, \mathbf{x}^{\prime \prime}\right) \in L_{p}\left(\Omega^{\prime}\right)$ for a. e. $\mathbf{x}^{\prime \prime} \in \mathbb{R}^{d_{2}}$, where $p=1$ for $d_{1}=1$ and $2 p>d_{1}$ for $d_{1} \geq 2$, and

$$
\underset{\mathbf{x}^{\prime \prime} \in \mathbb{R}^{d_{2}}}{\operatorname{ess} \sup }\left\|V\left(\cdot, \mathbf{x}^{\prime \prime}\right)\right\|_{L_{p}\left(\Omega^{\prime}\right)} \leq \widetilde{c}_{6}<\infty .
$$

We put

$$
\begin{gathered}
g_{j}(\mathbf{x})=\widetilde{g}_{j}(\mathbf{x}) \omega^{2}(\mathbf{x}), \quad j=1,2, \\
Q_{\lambda}(\mathbf{x})=\left(\lambda-V_{2}(\mathbf{x})\right) \omega^{2}(\mathbf{x}), \\
Q_{\lambda}^{0}\left(\mathbf{x}^{\prime \prime}\right)=\mid \Omega^{\prime-1} \int_{\Omega^{\prime}} Q_{\lambda}\left(\mathbf{x}^{\prime}, \mathbf{x}^{\prime \prime}\right) d \mathbf{x}^{\prime} .
\end{gathered}
$$

Suppose that $\lambda$ is such that

$$
\lambda \omega_{0}^{2}-\omega_{1}^{2} \widetilde{c}_{4}>0 .
$$

For any $\Gamma$-periodic (in $\mathbf{x}^{\prime}$ ) function $\varphi$, we use the notation

$$
\varphi^{\varepsilon}(\mathbf{x})=\varphi\left(\varepsilon^{-1} \mathbf{x}^{\prime}, \mathbf{x}^{\prime \prime}\right) .
$$

In $L_{2}\left(\mathbb{R}^{d}\right)$, consider the Schrödinger operator

$$
H_{\varepsilon}=\mathbf{D}_{\mathbf{x}^{\prime}}^{*} \widetilde{g}_{1}^{\varepsilon} \mathbf{D}_{\mathbf{x}^{\prime}}+\mathbf{D}_{\mathbf{x}^{\prime \prime}}^{*} \widetilde{g}_{2}^{\varepsilon} \mathbf{D}_{\mathbf{x}^{\prime \prime}}+\varepsilon^{-2} V^{\varepsilon} .
$$


The precise definition of $H_{\varepsilon}$ is given in terms of the quadratic form

$$
h_{\varepsilon}[u, u]=\int_{\mathbb{R}^{d}}\left(\left\langle\widetilde{g}_{1}^{\varepsilon} \mathbf{D}_{\mathbf{x}^{\prime}} u, \mathbf{D}_{\mathbf{x}^{\prime}} u\right\rangle_{\mathbb{C}^{d_{1}}}+\left\langle\widetilde{g}_{2}^{\varepsilon} \mathbf{D}_{\mathbf{x}^{\prime \prime}} u, \mathbf{D}_{\mathbf{x}^{\prime \prime}} u\right\rangle_{\mathbb{C}^{d_{2}}}+\varepsilon^{-2} V^{\varepsilon}|u|^{2}\right) d \mathbf{x}, \quad u \in H^{1}\left(\mathbb{R}^{d}\right) .
$$

The following result can be deduced from Theorem 19 similarly to deduction of Theorem 18 from Theorem 1.

Theorem 17 Let $\widetilde{g}_{1}, \widetilde{g}_{2}$, $\omega$ be measurable functions in $\mathbb{R}^{d}$, periodic in $\mathbf{x}^{\prime}$ with respect to the lattice $\Gamma$ and satisfying conditions (10.13)-(10.16). Suppose that the functions $V, V_{2}$ defined by (10.17), (10.18) satisfy conditions (10.19)-(10.21). Let $H_{\varepsilon}$ be the operator in $L_{2}\left(\mathbb{R}^{d}\right)$ corresponding to the quadratic form (10.27). Let $A_{\varepsilon}$ be the operator (10.3) with the coefficients (10.22), and let $A^{0}$ be the corresponding effective operator (10.8) with coefficients defined according to (10.9), (10.10). Let $Q_{\lambda}$ be defined by (10.23), and let restriction (10.25) be satisfied. Let $Q_{\lambda}^{0}$ be given by (10.24). Then we have

$$
\left\|\left(H_{\varepsilon}+\lambda I\right)^{-1}-\omega^{\varepsilon}\left(A^{0}+Q_{\lambda}^{0}\right)^{-1} \omega^{\varepsilon}\right\|_{L_{2}\left(\mathbb{R}^{d}\right) \rightarrow L_{2}\left(\mathbb{R}^{d}\right)} \leq C_{\lambda} \varepsilon, \quad 0<\varepsilon \leq 1 .
$$

Here the constant $C_{\lambda}$ depends only on $\widetilde{c}_{0}, \widetilde{c}_{1}, \widetilde{c}_{2}, \omega_{0}, \omega_{1}, \widetilde{c}_{3}, \widetilde{c}_{4}, \widetilde{c}_{5}, \lambda$ and parameters of the lattice $\Gamma$.

Similarly to Subsection 10.4, it is possible to give sufficient conditions on $\widetilde{g}_{1}, \widetilde{g}_{2}, V$, which ensure representation (10.17) and conditions of Theorem 20. For this, consider the Schrödinger operator

$$
H\left(\mathbf{x}^{\prime \prime}\right)=\mathbf{D}_{\mathbf{x}^{\prime}}^{*} \widetilde{g}_{1}\left(\mathbf{x}^{\prime}, \mathbf{x}^{\prime \prime}\right) \mathbf{D}_{\mathbf{x}^{\prime}}+\varepsilon^{-2} V\left(\mathbf{x}^{\prime}, \mathbf{x}^{\prime \prime}\right),
$$

acting in $L_{2}\left(\mathbb{R}^{d_{1}}\right)$ and depending on parameter $\mathbf{x}^{\prime \prime} \in \mathbb{R}^{d_{2}}$. Assume that $\widetilde{g}_{j}, V$ are $\Gamma$-periodic in $\mathbf{x}^{\prime}$ and satisfy (10.13) and (10.21). Assume also that the bottom of the spectrum of the operator (10.29) coincides with point $\lambda=0$ :

$$
\inf \operatorname{spec} H\left(\mathbf{x}^{\prime \prime}\right)=0, \quad \text { a. e. } \mathbf{x}^{\prime \prime} \in \mathbb{R}^{d_{2}} .
$$

If condition (10.30) is satisfied, there exists a positive periodic (in $\left.\mathbf{x}^{\prime}\right)$ solution $\omega\left(\mathbf{x}^{\prime}, \mathbf{x}^{\prime \prime}\right)$ of the equation

$$
\mathbf{D}_{\mathbf{x}^{\prime}}^{*} \widetilde{g}_{1}\left(\mathbf{x}^{\prime}, \mathbf{x}^{\prime \prime}\right) \mathbf{D}_{\mathbf{x}^{\prime}} \omega\left(\mathbf{x}^{\prime}, \mathbf{x}^{\prime \prime}\right)+V\left(\mathbf{x}^{\prime}, \mathbf{x}^{\prime \prime}\right) \omega\left(\mathbf{x}^{\prime}, \mathbf{x}^{\prime \prime}\right)=0 .
$$

This solution can be fixed by the condition

$$
\int_{\Omega^{\prime}} \omega^{2}\left(\mathbf{x}^{\prime}, \mathbf{x}^{\prime \prime}\right) d \mathbf{x}^{\prime}=\left|\Omega^{\prime}\right|
$$

Moreover, this solution satisfies (10.15), where $\omega_{0}, \omega_{1}$ are controlled in terms of $\widetilde{c}_{0}, \widetilde{c}_{1}, \widetilde{c}_{6}$. Concerning the properties of solution $\omega$, see [KiSi] and $\S 4$ of [BSu5]. By (10.31), representation (10.17) is true.

Next, it is possible to ensure conditions (10.16), (10.19) and (10.20) imposing some smoothness assumptions on coefficients $\widetilde{g}_{j}, V$ with respect to $\mathbf{x}^{\prime \prime}$. It suffices to assume that derivatives $D_{\mathbf{x}^{\prime \prime}}^{\alpha} \widetilde{g}_{1}$, $|\alpha| \leq 3$, are uniformly bounded, derivatives $D_{\mathbf{x}^{\prime \prime}}^{\alpha} \widetilde{g}_{2},|\alpha| \leq 2$, are uniformly bounded and that the norms $\left\|D_{\mathbf{x}^{\prime \prime}}^{\alpha} V\left(\cdot, \mathbf{x}^{\prime \prime}\right)\right\|_{L_{p}\left(\Omega^{\prime}\right)},|\alpha| \leq 3$, are uniformly bounded.

Under the above assumptions, all conditions of Theorem 20 are satisfied.

\section{References}

[BaPa] Bakhvalov N. S., Panasenko G. P., Homogenization: averaging processes in periodic media. Mathematical problems in mechanics of composite materials, "Nauka", Moscow, 1984; English transl., Math. Appl. (Soviet Ser.), vol. 36, Kluwer Acad. Publ. Group, Dordrecht, 1989.

[BeLP] Bensoussan A., Lions J. L., Papanicolaou G., Asymptotic analysis for periodic structures, Stud. Math. Appl., vol. 5, North-Holland Publishing Co., Amsterdam-New York, 1978. 
[BSu1] Birman M. Sh., Suslina T. A., Second order periodic differential operators. Threshold properties and homogenization, Algebra i Analiz 15 (2003), no. 5, 1-108; Engl. transl., St. Petersburg Math. J. 15 (2004), no. 5, 639-714.

[BSu2] Birman M. Sh., Suslina T. A., Threshold approximations with corrector for the resolvent of a factorized selfadjoint operator family, Algebra i Analiz 17 (2005), no. 5, 69-90; English transl., St. Petersburg Math. J. 17 (2006), no. 5, 745-762.

[BSu3] Birman M. Sh., Suslina T. A., Homogenization with corrector term for periodic elliptic differential operators, Algebra i Analiz 17 (2005), no. 6, 1-104; English transl., St. Petersburg Math. J. 17 (2006), no. 6, 897-974.

[BSu4] Birman M. Sh., Suslina T. A., Homogenization with corrector term for periodic differential operators. Approximation of solutions in Sobolev class $H^{1}(\mathbb{R})$, Algebra i Analiz 18 (2006), no. 6, 1-130; English transl., St. Petersburg Math. J. 18 (2007), no. 6.

[BSu5] Birman M. Sh., Suslina T. A., Two-dimensional periodic Pauli operator. The effective masses at the lower edge of the spectrum, Oper. Theory: Adv. Appl., Vol. 108, 1999, Birkhäuser, Basel.

[CoV] Conca C., Vanninathan M., Homogenization of periodic structures via Bloch decomposition, SIAM J. Appl. Math. 57 (1997), no. 6, 1639-1659.

[GT] Gilbarg D., Trudinger N. S., Elliptic partial differential equations of second order, Grundlehren der mathematischen Wissenschaften, 224, Berlin-Heidelberg-New York: Springer-Verlag (1977).

[FK] Filonov N., Klopp F., Absolute continuity of the spectrum of a Schrödinger operator with a potential which is periodic in some directions and decays in others, Doc. Math. 9 (2004), 107-121.

[Zh] Zhikov V. V., Spectral approach to asymptotic diffusion problems, Differentsial'nye Uravneniya 25 (1989), no. 1, 44-50; English transl., Differential Equations 25 (1989), no. 1, 33-39.

[ZhKO] Zhikov V. V., Kozlov S. M., Olejnik O. A., Homogenization of differential operators, Fizmatgiz, Moscow, 1993; Engl. transl., Springer-Verlag, Berlin, 1994.

[KiSi] Kirsch W, Simon B., Comparison theorems for the gap of Schrödinger operators, J. Funct. Anal. 75 (1987), no. 2, 396-410.

[Sa] Sanchez-Palencia E., Nonhomogeneous media and vibration theory, Lecture Notes in Phys., vol. 127, Springer-Verlag, Berlin-New York, 1980.

[Se] Sevost'yanova E. V., Asymptotic expansion of the solution of a second-order elliptic equation with periodic rapidly oscillating coefficients, Mat. Sb. (N.S.) 115 (1981), no. 2, 204-222; English transl., Math. USSR-Sb. 43 (1982), no. 2, 181-198.

[Su] Suslina T. A., On homogenization for a periodic elliptic operator in a strip, Algebra i Analiz 16 (2004), no. 1, 269-292; Engl. transl.: St. Petersburg Math. J. 16 (2004), no. 1, 237-257. 